\title{
On the Mean Residual Life Function and Stress and Strength Analysis under Different Loss Function for Lindley Distribution
}

\author{
Sajid Ali \\ Department of Decision Sciences, Bocconi University, via Roenthen 1, 20136 Milan, Italy \\ Correspondence should be addressed to Sajid Ali; sajidali.qau@hotmail.com
}

Received 4 November 2012; Accepted 4 February 2013

Academic Editor: Shey-Huei Sheu

Copyright (C) 2013 Sajid Ali. This is an open access article distributed under the Creative Commons Attribution License, which permits unrestricted use, distribution, and reproduction in any medium, provided the original work is properly cited.

\begin{abstract}
Purpose. Mathematical properties of Lindley distribution are derived under different loss functions. These properties include mean residual life function, Lorenz curve, stress and strength characteristic, and their respective posterior risk via simulation scheme. Methodology. Bayesian approach is used for the reliability characteristics. Results are compared on the basis of posterior risk. Findings. Using prior information on the parameter of Lindley distribution, Bayes estimates for reliability characteristics are compared under different loss functions. Practical Implications. Since Lindley distribution is a mixture of gamma and exponential distribution, so Bayesian estimation of reliability characteristics will have a great implication in reliability theory. Originality. A real life application to waiting time data at the bank is also described for the developed procedures. This study is useful for researcher and practitioner in reliability theory.
\end{abstract}

\section{Introduction}

Exponential distribution is frequently used as a lifetime distribution in statistics and applied areas; the Lindley distribution has been ignored in the literature since 1958. Lindley distribution originally developed by Lindley [1] and some classical statistic properties are investigated by Ghitany et al. [2]. Sankaran [3] introduced a discrete version of Lindley distribution known as discrete Poisson-Lindley distribution, and Ghitany and Al-Mutairi [4] described some estimation methods. The distribution of zero-truncated Poisson-Lindley was introduced by Ghitany et al. [5] who used the distribution for modeling count data in the case where the distribution has to be adjusted for the count of missing zeros. Zamani and Ismail [6] introduced negative binomial distribution as an alternative to zero-truncated Poisson-Lindley distribution. Recently, Ghitany et al. [7] introduced a two-parameter weighted Lindley distribution and pointed that Lindley distribution is particularly useful in modelling biological data from mortality studies.

The rest of the study is organized as follows. Section 2 deals with the derivation of posterior distribution using different noninformative and informative priors. Using different loss functions, the Bayes estimators and their respective posterior risks are discussed in Section 3. Elicitation of hyperparameter is also discussed in Section 3. Simulation study of Bayes estimates of mean residual life and their posterior risks is performed in Section 4. Lorenz curve discussion for Lindley distribution is given in Section 5 while stress and strength reliability characteristics and simulation study under different loss functions is discussed/performed in Section 6. Real life application is illustrated in Section 7. Finally, Section 8 deals with a conclusion and some future remarks.

\section{Likelihood Function and Posterior Distributions}

The posterior distribution summarizes available probabilistic information on the parameters in the form of prior distribution and the sample information contained in the likelihood function. The likelihood principle suggests that the information on the parameter should depend only on its posterior distribution. Bayesian scientist's job is to assist the investigator to extract features of interest from the posterior distribution. In this section, we will use the Lindley model as sampling distribution mingles with noninformative priors for the derivation of posterior distribution. A random variable $X$ 
TABLE 1: Bayes estimator and posterior risk under different loss functions.

\begin{tabular}{lcr}
\hline Loss function & Bayes estimator (BE) & Posterior risk (PR) \\
\hline$L_{1}=\operatorname{SELF}=(\theta-d)^{2}$ & $E(\theta \mid \mathbf{x})$ & $\operatorname{Var}(\theta \mid \mathbf{x})$ \\
$L_{2}=$ WSELF $=\frac{(\theta-d)^{2}}{\theta}$ & $\left(E\left(\theta^{-1} \mid \mathbf{x}\right)\right)^{-1}$ & $E(\theta \mid \mathbf{x})-\left(E\left(\theta^{-1} \mid \mathbf{x}\right)\right)^{-1}$ \\
$L_{3}=$ M/Q SELF $=\left(1-\frac{d}{\theta}\right)^{2}$ & $\frac{E\left(\theta^{-1} \mid \mathbf{x}\right)}{E\left(\theta^{-2} \mid \mathbf{x}\right)}$ & $1-\frac{E\left(\theta^{-1} \mid \mathbf{x}\right)^{2}}{E\left(\theta^{-2} \mid \mathbf{x}\right)}$ \\
$L_{4}=$ PLF $=\frac{(\theta-d)^{2}}{d}$ & $\sqrt{E\left(\theta^{2} \mid \mathbf{x}\right)}$ & $2\left[\sqrt{E\left(\theta^{2} \mid \mathbf{x}\right)}-E(\theta \mid \mathbf{x})\right]$ \\
$L_{5}=\operatorname{SLLF}=(\log \theta-\log d)^{2}$ & $\exp (E(\log \theta \mid \mathbf{x}))$ & $\operatorname{Var}(\log \theta \mid \mathbf{x})$ \\
$L_{6}=\mathrm{ELF}=\left[\frac{d}{\theta}-\log \frac{d}{\theta}-1\right]$ & $\left(E\left(\theta^{-1} \mid \mathbf{x}\right)\right)^{-1}$ & $2\left[E(\theta \mid \mathbf{x})-\log \left(E\left(\theta^{-1} \mid \mathbf{x}\right)\right)\right.$ \\
$L_{7}=\mathrm{KLF}=\left(\sqrt{\frac{d}{\theta}}-\sqrt{\frac{\theta}{d}}\right)^{2}$ & $\sqrt{\frac{E(\theta \mid \mathbf{x})}{E\left(\theta^{-1} \mid \mathbf{x}\right)}}$ & \\
\hline
\end{tabular}

TABLE 2: BEs and their respective PRs of MRLF under SELF.

\begin{tabular}{|c|c|c|c|c|c|c|}
\hline$n$ & & UP & & & JP & \\
\hline$\theta$ & 7.142857 & 1.2 & 0.114114 & 7.142857 & 1.2 & 0.114114 \\
\hline 20 & 16.6609 (8.74099) & $1.16041(0.049399)$ & $0.108763(0.000527)$ & $17.1312(8.650769)$ & $1.2008(0.047705)$ & $0.113795(0.000504)$ \\
\hline 40 & $16.9256(4.50602)$ & $1.17773(0.025448)$ & $0.111713(0.000277)$ & $17.1644(4.44596)$ & $1.1982(0.025012)$ & $0.11429(0.000271)$ \\
\hline 60 & $16.9761(3.02169)$ & $1.18603(0.017205)$ & $0.112294(0.000186)$ & $17.1357(2.99484)$ & $1.19976(0.017109)$ & $0.11402(0.000184)$ \\
\hline 80 & $17.0298(2.28011)$ & 1.19015 (0.012994) & $0.112893(0.000141)$ & $17.1499(2.26492)$ & $1.20048(0.012883)$ & $0.114194(0.000139)$ \\
\hline 100 & $17.0348(1.82524)$ & $1.19247(0.010436)$ & $0.113075(0.000113)$ & $17.1309(1.81551)$ & $1.20075(0.010364)$ & $0.114117(0.000112)$ \\
\hline$n$ & & LMP & & & GP & \\
\hline$\theta$ & 7.142857 & 1.2 & 0.114114 & 7.142857 & 1.2 & 0.114114 \\
\hline 20 & $16.6296(8.574081)$ & $1.15994(0.044900)$ & $0.110424(0.000504)$ & $17.1139(8.52409)$ & $1.19828(0.045907)$ & $0.115161(0.000489)$ \\
\hline 40 & $16.9097(4.40598)$ & 1.17747 (0.025014) & $0.112539(0.000270)$ & $17.1556(4.40518)$ & $1.19692(0.025013)$ & $0.114963(0.000266)$ \\
\hline 60 & $16.9654(2.903167)$ & $1.18586(0.017105)$ & $0.112844(0.000185)$ & $17.1298(2.906730)$ & $1.19891(0.017032)$ & $0.114467(0.000182)$ \\
\hline 80 & $17.0218(2.25801)$ & $1.19002(0.012794)$ & $0.113305(0.000140)$ & $17.1455(2.25698)$ & $1.19984(0.012796)$ & $0.114528(0.000139)$ \\
\hline 100 & $17.0284(1.81523)$ & $1.19236(0.010354)$ & $0.113405(0.000113)$ & $17.1274(1.81519)$ & $1.20023(0.010353)$ & $0.114385(0.000112)$ \\
\hline
\end{tabular}

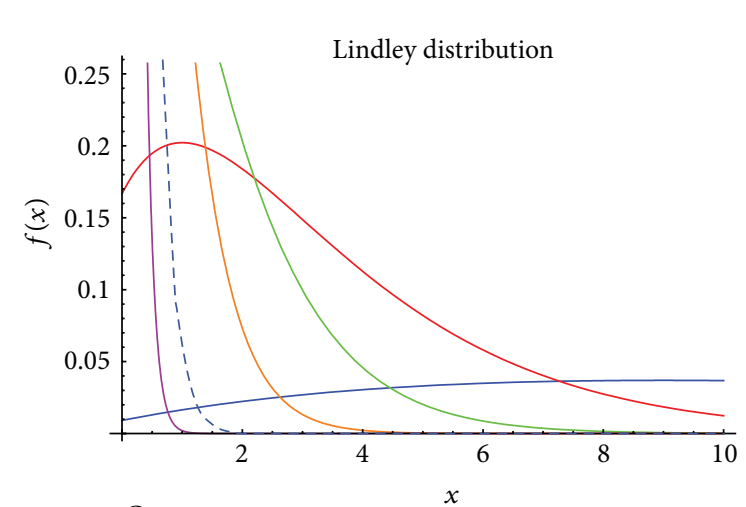

$\begin{array}{rlrl}\text { Curve } & & x \\ -\theta & =0.1 & -\theta & =2 \\ -\theta & =0.5 & --\theta & =5 \\ -\theta & =1 & -\theta & =9\end{array}$

Figure 1: The Lindley distribution.

is said to possess a Lindley distribution if it has the following form:

$$
f(x)=\frac{\theta^{2}}{\theta+1}(1+x) e^{-\theta x}, \quad x, \theta>0
$$

It is obvious from Figure 1 that behavior of Lindley distribution is close to exponential or gamma distribution.

The likelihood function for a random sample $x_{1}, x_{2}, \ldots, x_{n}$ which is taken from Lindley distribution is

$$
L(\mathbf{x}, \theta)=\frac{\theta^{2 n}}{(\theta+1)^{n}} \prod_{i=1}^{n}\left(1+x_{i}\right) e^{-\theta \sum_{i=1}^{n} x_{i}}, \quad \theta>0
$$

\subsection{Posterior Distribution Using Noninformative Priors}

2.1.1. Uniform Prior. An argument in the favor of uniform prior is that when the data are sufficiently informative, so that likelihood function is sharply peaked, then it really does not matter what prior is used, since all reasonably smooth prior densities will lead to approximately the same posterior density. The uniform density in most cases is convenient to simplify calculations of the posterior. This argument supports the uniform prior only in those cases where it produces approximately the same conclusions as the highly imprecise prior constructed from a sufficiently large class of prior densities. If the data are highly informative, the uniform prior 
TABLE 3: BEs and their respective PRs of MRLF under WSELF.

\begin{tabular}{|c|c|c|c|c|c|c|}
\hline$n$ & & UP & & & $\mathrm{JP}$ & \\
\hline$\theta$ & 7.142857 & 1.2 & 0.114114 & 7.142857 & 1.2 & 0.114114 \\
\hline 20 & $16.1511(0.509768)$ & $1.11927(0.041144)$ & $0.10415(0.004613)$ & $16.6062(0.504952)$ & $1.1582(0.040598)$ & $0.10896(0.004538)$ \\
\hline 40 & $16.6632(0.262396)$ & $1.15649(0.021239)$ & $0.109296(0.002417)$ & $16.8981(0.262306)$ & $1.17658(0.021156)$ & $0.111815(0.002415)$ \\
\hline 60 & $16.7998(0.176282)$ & $1.17169(0.014341)$ & $0.110663(0.001631)$ & $16.9577(0.176031)$ & $1.18525(0.014309)$ & $0.112362(0.001627)$ \\
\hline 80 & $16.8969(0.132920)$ & $1.17933(0.010824)$ & $0.111659(0.001234)$ & 17.0159 (0.132909) & $1.18956(0.010819)$ & $0.112945(0.001234)$ \\
\hline 100 & $16.9283(0.106526)$ & $1.18378(0.008691)$ & $0.112084(0.000991)$ & $17.0238(0.105716)$ & $1.1920(0.008573)$ & $0.113117(0.000990)$ \\
\hline$n$ & & LMP & & & GP & \\
\hline$\theta$ & 7.142857 & 1.2 & 0.114114 & 7.142857 & 1.2 & 0.114114 \\
\hline 20 & $16.1217(0.504878)$ & $1.11883(0.040112)$ & $0.105691(0.004437)$ & $16.5891(0.504848)$ & $1.15569(0.042586)$ & $0.110343(0.004418)$ \\
\hline 40 & $16.6478(0.261905)$ & $1.15624(0.021123)$ & $0.110091(0.002414)$ & $16.8893(0.261276)$ & $1.17531(0.021111)$ & $0.112492(0.002417)$ \\
\hline 60 & $16.7894(0.176031)$ & $1.17152(0.014307)$ & $0.111199(0.001624)$ & $16.9518(0.176019)$ & $1.18440(0.014308)$ & $0.112811(0.001625)$ \\
\hline 80 & $16.889(0.132795)$ & $1.1792(0.010822)$ & $0.112063(0.001232)$ & $17.0115(0.132902)$ & $1.18892(0.010819)$ & $0.11328(0.001228)$ \\
\hline 100 & $16.9220(0.105446)$ & $1.18367(0.008489)$ & $0.112409(0.000991)$ & $17.0202(0.105156)$ & $1.19148(0.008452)$ & $0.113385(0.000990)$ \\
\hline
\end{tabular}

TABLE 4: BEs and their respective PRs of MRLF under MSELF.

\begin{tabular}{|c|c|c|c|c|c|c|}
\hline$n$ & & UP & & & $\mathrm{JP}$ & \\
\hline$\theta$ & 7.142857 & 1.2 & 0.114114 & 7.142857 & 1.2 & 0.114114 \\
\hline 20 & $15.6991(3979.6)$ & $1.0835(0.313983)$ & $0.100281(0.998953)$ & $16.1011(3504.08)$ & $1.11726(0.314576)$ & $0.104331(0.998814)$ \\
\hline 40 & $16.4163(4489.67)$ & $1.13671(0.494297)$ & $0.107086(0.998747)$ & $16.6369(4476.16)$ & $1.1554(0.506637)$ & $0.109393(0.998662)$ \\
\hline 60 & $16.6305(4645.4)$ & $1.15802(0.571236)$ & $0.109126(0.998682)$ & $16.7819(4477.83)$ & $1.17094(0.562509)$ & $0.110729(0.998622)$ \\
\hline 80 & $16.7679(4749.79)$ & $1.16888(0.611298)$ & $0.110479(0.998637)$ & $16.8833(4749.34)$ & $1.17875(0.625842)$ & 0.111709 (0.998591) \\
\hline 100 & $16.8244(4790.7)$ & $1.17533(0.635275)$ & $0.111128(0.998616)$ & $16.9174(4718.19)$ & $1.18331(0.609666)$ & $0.112125(0.998578)$ \\
\hline$n$ & & LMP & & & GP & \\
\hline$\theta$ & 7.142857 & 1.2 & 0.114114 & 7.142857 & 1.2 & 0.114114 \\
\hline 20 & $15.6738(3509.61)$ & $1.08313(0.312572)$ & $0.101543(0.99881)$ & $16.0855(3491.33)$ & $1.11502(0.306844)$ & $0.105556(0.998771)$ \\
\hline 40 & $16.4021(4477.74)$ & $1.13648(0.493397)$ & $0.107804(0.998572)$ & $16.6286(4469.05)$ & $1.15419(0.50569)$ & $0.11003(0.998638)$ \\
\hline 60 & $16.6207(4437.01)$ & $1.15786(0.560576)$ & $0.109627(0.998624)$ & $16.7763(4369.97)$ & $1.17011(0.52163)$ & $0.11116(0.998606)$ \\
\hline 80 & $16.7604(4743.29)$ & $1.16876(0.610779)$ & $0.110863(0.998523)$ & $16.8790(4745.63)$ & $1.17813(0.610592)$ & $0.112035(0.998528)$ \\
\hline 100 & $16.8182(4785.43)$ & $1.17523(0.604848)$ & $0.11144(0.998573)$ & $16.9140(4768.19)$ & $1.18281(0.596919)$ & $0.112386(0.998568)$ \\
\hline
\end{tabular}

may produce reasonable inferences. The uniform prior for $\theta$ is defined as

$$
p(\theta) \propto 1, \quad \theta>0
$$

The posterior distribution of parameter $\theta$ for the given data using (2) and (3) is

$$
p(\theta \mid \mathbf{x})=\frac{\left(\left(\theta^{2 n}\right) /(\theta+1)^{n}\right) e^{-\theta \sum_{i=1}^{n} x_{i}}}{\left(\left(\theta^{2 n}\right) /(\theta+1)^{n}\right) e^{-\theta \sum_{i=1}^{n} x_{i}} d \theta}, \quad \theta>0 .
$$

2.1.2. Jeffreys Prior. Jeffreys was motivated by invariance requirements and suggested a solution to provide a noninformative prior. He used differential geometry method. The requirements are invariance under 1-1 transformations and invariance under sufficient statistics. One-dimensional version of Jeffreys prior has been justified from many different viewpoints. Jeffreys [8] proposed a formal rule for obtaining a noninformative prior as follows. If $\theta$ is a $\mathrm{k}$-vector valued parameter, then, JP of $\theta$ is $g(\theta) \propto \sqrt{|\operatorname{det} I(\theta)|}$ where $I(\theta)$ is a kxk Fisher's (information) matrix whose $(i, j)$ th element is $-E\left[\partial^{2} \ln L(\theta \mid \mathbf{x}) / \partial \theta_{i} \partial \theta_{j}\right] i, j=1,2, \ldots, k$. Fisher's information matrix is not directly related to the notation of lack of information. The connection comes from the role of Fisher's matrix in asymptotic theory. Jeffreys noninformative priors based on Fisher's information matrix often lead to a family of improper priors. The Jeffreys prior of the parameter $\theta$ is

$$
\begin{gathered}
p(\theta) \propto \frac{\sqrt{\theta^{2}+4 \theta+2}}{\theta(1+\theta)}, \quad \theta>0, \\
p(\theta \mid x) \\
=\frac{\left(\left(\theta^{2 n-1}\right) /(\theta+1)^{n+1}\right) \sqrt{\theta^{2}+4 \theta+2} e^{-\theta \sum_{i=1}^{n} x_{i}}}{\int_{0}^{\infty}\left(\left(\theta^{2 n-1}\right) /(\theta+1)^{n+1}\right) \sqrt{\theta^{2}+4 \theta+2} e^{-\theta \sum_{i=1}^{n} x_{i}} d \theta}, \quad \theta>0 .
\end{gathered}
$$

2.2. Posterior Distribution Using Informative Prior. In case of an informative prior, the use of prior information is equivalent to adding a number of observations to a given sample size and, therefore, leads to a reduction of the variance/posterior 
TABLE 5: BEs and their respective PRs of MRLF under PLF.

\begin{tabular}{|c|c|c|c|c|c|c|}
\hline$n$ & & UP & & & $\mathrm{JP}$ & \\
\hline$\theta$ & 7.142857 & 1.2 & 0.114114 & 7.142857 & 1.2 & 0.114114 \\
\hline 20 & $16.9211(0.520576)$ & $1.1815(0.042188)$ & $0.111158(0.004791)$ & $17.3777(0.493072)$ & $1.2205(0.039405)$ & $0.115987(0.004383)$ \\
\hline 40 & $17.0582(0.265186)$ & $1.18848(0.021509)$ & $0.112945(0.002464)$ & $17.2934(0.258053)$ & $1.20859(0.020784)$ & $0.115468(0.002356)$ \\
\hline 60 & $17.0648(0.177533)$ & $1.19326(0.014463)$ & $0.11312(0.001653)$ & $17.2229(0.174329)$ & $1.20683(0.012135)$ & $0.114822(0.001604)$ \\
\hline 80 & $17.0966(0.133627)$ & $1.1956(0.010893)$ & $0.113516(0.001246)$ & $17.2158(0.131813)$ & $1.20584(0.010708)$ & $0.114803(0.001218)$ \\
\hline 100 & $17.0883(0.10698)$ & $1.19684(0.008735)$ & $0.113575(0.000999)$ & $17.1838(0.105815)$ & $1.20506(0.008616)$ & $0.114608(0.000981)$ \\
\hline$n$ & & LMP & & & GP & \\
\hline$\theta$ & 7.142857 & 1.2 & 0.114114 & 7.142857 & 1.2 & 0.114114 \\
\hline 20 & $16.8904(0.485218)$ & $1.18104(0.034205)$ & $0.112775(0.004302)$ & $17.3612(0.492507)$ & $1.21811(0.033652)$ & $0.117262(0.004203)$ \\
\hline 40 & $17.0425(0.256431)$ & $1.18823(0.020514)$ & $0.11376(0.002342)$ & $17.2848(0.256428)$ & $1.20734(0.020449)$ & $0.116118(0.002310)$ \\
\hline 60 & $17.0543(0.173642)$ & $1.19309(0.011465)$ & $0.113666(0.001602)$ & $17.2171(0.173498)$ & $1.20599(0.011464)$ & $0.115259(0.001583)$ \\
\hline 80 & $17.0886(0.131689)$ & $1.19547(0.010704)$ & $0.113926(0.001214)$ & $17.2114(0.131609)$ & $1.20520(0.010704)$ & $0.115132(0.001207)$ \\
\hline 100 & 17.0819 (0.105019) & $1.19673(0.008536)$ & $0.113903(0.000979)$ & $17.1803(0.105017)$ & $1.20454(0.008527)$ & $0.114871(0.0009735)$ \\
\hline
\end{tabular}

TABLE 6: BEs and their respective PRs of MRLF under SLLF.

\begin{tabular}{|c|c|c|c|c|c|c|}
\hline$n$ & & UP & & & JP & \\
\hline$\theta$ & 7.142857 & 1.2 & 0.114114 & 7.142857 & 1.2 & 0.114114 \\
\hline 20 & $16.4002(0.031314)$ & $1.13929(0.036423)$ & $0.106377(0.043796)$ & $16.8701(0.031313)$ & $1.17967(0.036422)$ & $0.111405(0.043784)$ \\
\hline 40 & $16.7929(0.015685)$ & $1.16697(0.018282)$ & $0.110483(0.021993)$ & $17.0316(0.015687)$ & $1.18743(0.018286)$ & $0.113059(0.021026)$ \\
\hline 60 & $16.8873(0.010466)$ & $1.1788(0.012203)$ & $0.111469(0.014688)$ & $17.0468(0.010471)$ & $1.19253(0.012205)$ & $0.113194(0.014687)$ \\
\hline 80 & $16.9629(0.007851)$ & $1.1847(0.009157)$ & $0.112271(0.011023)$ & $17.083(0.007854)$ & $1.19503(0.009158)$ & $0.113571(0.011024)$ \\
\hline 100 & $16.9813(0.006283)$ & $1.1881(0.007329)$ & $0.112576(0.008823)$ & $17.0774(0.006282)$ & $1.19638(0.007329)$ & $0.113618(0.008826)$ \\
\hline$n$ & & LMP & & & GP & \\
\hline$\theta$ & 7.142857 & 1.2 & 0.114114 & 7.142857 & 1.2 & 0.114114 \\
\hline 20 & $16.3694(0.031254)$ & $1.13883(0.036408)$ & $0.108011(0.043296)$ & $16.8526(0.031289)$ & $1.17711(0.036412)$ & $0.112809(0.043172)$ \\
\hline 40 & $16.7772(0.015670)$ & $1.16672(0.018277)$ & $0.111303(0.021016)$ & $17.0228(0.015674)$ & $1.18614(0.018204)$ & $0.113742(0.021026)$ \\
\hline 60 & $16.8767(0.010459)$ & $1.17862(0.012201)$ & $0.112016(0.014542)$ & $17.0410(0.010454)$ & $1.19166(0.012202)$ & $0.113645(0.014523)$ \\
\hline 80 & $16.955(0.007847)$ & $1.18457(0.009156)$ & $0.112681(0.011023)$ & $17.0786(0.007845)$ & $1.19438(0.009153)$ & $0.113908(0.010987)$ \\
\hline 100 & $16.9749(0.006280)$ & $1.18799(0.007328)$ & $0.112905(0.008822)$ & $17.0739(0.006286)$ & $1.19586(0.007329)$ & $0.113887(0.008800)$ \\
\hline
\end{tabular}

risk of the Bayes estimates. Bansal [9] discussed a method to evaluate the relevance of a prior information in terms of the number of additional observation supposed to be added to a given sample size. We used the gamma and conjugate informative prior for analysis.

2.2.1. Gamma Prior. Posterior distribution using informative gamma prior (GP) is

$$
\begin{aligned}
& p(\theta \mid x) \\
& \quad=\frac{\left(\left(\theta^{2 n+a-1}\right) /(\theta+1)^{n}\right) e^{-\theta\left(\sum_{i=1}^{n} x_{i}+b\right)}}{\int_{0}^{\infty}\left(\left(\theta^{2 n+a-1}\right) /(\theta+1)^{n}\right) e^{-\theta\left(\sum_{i=1}^{n} x_{i}+b\right)} d \theta}, \quad a, b, \theta>0 .
\end{aligned}
$$

2.2.2. Likelihood Matching Prior (Conjugate Prior). The likelihood matching prior (LMP) for Lindley distribution is

$$
p(\theta) \propto \frac{\theta^{2 a}}{(\theta+1)^{a}} e^{-\theta b}, \quad a, b, \theta>0,
$$

and the posterior distribution using LMP is

$$
\begin{aligned}
& p(\theta \mid x) \\
& =\frac{\left(\left(\theta^{2 n+2 a}\right) /(\theta+1)^{n+a}\right) e^{-\theta\left(\sum_{i=1}^{n} x_{i}+b\right)}}{\int_{0}^{\infty}\left(\left(\theta^{2 n+a-1}\right) /(\theta+1)^{n}\right) e^{-\theta\left(\sum_{i=1}^{n} x_{i}+b\right)} d \theta}, \quad a, b, \theta>0 .
\end{aligned}
$$

\section{Bayes Estimators and Posterior Risk under Different Loss Functions}

This section spotlight is on the derivation of the Bayes estimator (BE) under different loss functions and their respective posterior risk (PR). The results are compared for noninformative as well as informative priors. If the decision is a choice of an estimator, then, the Bayes decision is a Bayes estimator. The Bayes estimators are evaluated under squared error loss function (SELF), weighted squared error loss function (WSELF), precautionary loss function (PLF), modified (quadratic) squared error loss function (M/Q SELF), logarithmic loss function (SLLF), entropy loss function (ELF), 
TABLE 7: BEs and their respective PRs of MRLF under ELF.

\begin{tabular}{|c|c|c|c|c|c|c|}
\hline$n$ & & UP & & & $\mathrm{JP}$ & \\
\hline$\theta$ & 7.142857 & 1.2 & 0.114114 & 7.142857 & 1.2 & 0.114114 \\
\hline 20 & $16.1511(5.57928)$ & $1.11927(0.243079)$ & $0.10415(0.150269)$ & $16.6062(5.53632)$ & $1.1582(0.231109)$ & $0.10896(0.150136)$ \\
\hline 40 & $16.6632(5.63416)$ & $1.15649(0.299794)$ & $0.109296(0.141658)$ & $16.8981(5.62276)$ & $1.17658(0.254403)$ & $0.111815(0.141275)$ \\
\hline 60 & $16.7998(5.64793)$ & $1.17169(0.322941)$ & $0.110663(0.139528)$ & $16.9577(5.64668)$ & $1.18525(0.326034)$ & $0.112362(0.136368)$ \\
\hline 80 & $16.8969(5.65816)$ & $1.17933(0.334435)$ & 0.111659 (0.137915) & $17.0159(5.62723)$ & $1.18956(0.315758)$ & $0.112945(0.136815)$ \\
\hline 100 & $16.9283(5.6611)$ & $1.18378(0.341065)$ & $0.112084(0.127363)$ & $17.0238(5.67237)$ & $1.1920(0.324957)$ & $0.113117(0.12645)$ \\
\hline$n$ & & LMP & & & GP & \\
\hline$\theta$ & 7.142857 & 1.2 & 0.114114 & 7.142857 & 1.2 & 0.114114 \\
\hline 20 & 16.1217 (5.53559) & $1.11883(0.22428)$ & $0.105691(0.147276)$ & $16.5891(5.53325)$ & $1.15569(0.207762)$ & $0.110343(0.148622)$ \\
\hline 40 & $16.6478(5.6233)$ & $1.15624(0.249369)$ & $0.110091(0.149015)$ & $16.8893(5.62316)$ & $1.17531(0.232234)$ & $0.112492(0.137587)$ \\
\hline 60 & $16.7894(5.64668)$ & $1.17152(0.322650)$ & $0.111199(0.148555)$ & $16.9518(5.61599)$ & $1.1844(0.314585)$ & $0.112811(0.145671)$ \\
\hline 80 & $16.889(5.62725)$ & $1.1792(0.314213)$ & $0.112063(0.138788)$ & 17.0115 (5.62175) & $1.18892(0.310674)$ & $0.11328(0.139026)$ \\
\hline 100 & $16.9220(5.66035)$ & $1.18367(0.320887)$ & $0.112409(0.128663)$ & $17.0202(5.65197)$ & $1.19148(0.35406)$ & $0.113385(0.124952)$ \\
\hline
\end{tabular}

TABLE 8: BEs and their respective PRs of MRLF under KLF.

\begin{tabular}{|c|c|c|c|c|c|c|}
\hline$n$ & & UP & & & JP & \\
\hline$\theta$ & 7.142857 & 1.2 & 0.114114 & 7.142857 & 1.2 & 0.114114 \\
\hline 20 & $16.404(0.063125)$ & $1.13965(0.073520)$ & $0.106432(0.088576)$ & $16.8667(0.063124)$ & $1.17931(0.073519)$ & $0.111351(0.088753)$ \\
\hline 40 & $16.7939(0.031494)$ & $1.16706(0.036732)$ & $0.110498(0.044231)$ & $17.0307(0.031159)$ & $1.18734(0.036731)$ & $0.113046(0.044226)$ \\
\hline 60 & $16.8877(0.020986)$ & $1.17884(0.024480)$ & $0.111475(0.029485)$ & $17.0464(0.020987)$ & $1.19249(0.024481)$ & $0.113188(0.029405)$ \\
\hline 80 & $16.9632(0.015733)$ & $1.18473(0.018357)$ & $0.112274(0.022107)$ & $17.0828(0.015732)$ & $1.19501(0.018359)$ & $0.113568(0.022108)$ \\
\hline 100 & $16.9815(0.012586)$ & $1.18811(0.014684)$ & $0.112579(0.017685)$ & $17.0773(0.012585)$ & $1.19636(0.014685)$ & $0.113616(0.017682)$ \\
\hline$n$ & & LMP & & & GP & \\
\hline$\theta$ & 7.142857 & 1.2 & 0.114114 & 7.142857 & 1.2 & 0.114114 \\
\hline 20 & $16.3737(0.063005)$ & $1.1392(0.073491)$ & $0.108032(0.088577)$ & $16.8494(0.063076)$ & $1.17679(0.073498)$ & $0.112726(0.087329)$ \\
\hline 40 & $16.7783(0.031144)$ & $1.16681(0.036724)$ & $0.111309(0.044177)$ & $17.0220(0.031132)$ & $1.18606(0.036726)$ & $0.113721(0.043938)$ \\
\hline 60 & $16.8772(0.020973)$ & $1.17867(0.024476)$ & $0.112019(0.029403)$ & $17.0406(0.020103)$ & $1.19163(0.024469)$ & $0.113636(0.029356)$ \\
\hline 80 & $16.9553(0.015725)$ & $1.1846(0.018355)$ & $0.112683(0.022108)$ & $17.0784(0.015723)$ & $1.19436(0.018358)$ & $0.113902(0.022035)$ \\
\hline 100 & $16.9751(0.012581)$ & $1.18801(0.014683)$ & $0.112906(0.017624)$ & $17.0737(0.012582)$ & $1.19585(0.014681)$ & $0.113883(0.017639)$ \\
\hline
\end{tabular}

and K-Loss function. K-loss function proposed by Wasan [10] is well fitted for a measure of inaccuracy for an estimator of a scale parameter of a distribution defined on $R^{+}=(0, \infty)$; this loss function is called K-loss function (KLF). Kanefuji and Iwase [11] used KLF for the estimation of a scale parameter with a known coefficient of variations. Table 1 (by [12]) will show the Bayes estimators and their posterior risks for the above-mentioned loss function.

3.1. Elicitation of Hyperparameter(s). Even though many authors have pointed a need for a formal and comprehensive process for elicitation of hyperparameters, there is no standard method. For elicitation, mainly two points are considered; the functional form of the prior distribution and hyperparameter(s), that is why a natural conjugate prior distribution has been generally recommended because its functional form is identical to likelihood function and posterior distribution can be determined by the way of conjugancy. To determine hyperparameter, we adopted the method discussed by Ali et al. [12].

\section{Mean Residual Life Function}

For a continuous distribution with the density $f(x)$ and cumulative distribution function $F(x)$, the mean residual life function is defined as

$$
m(x)=E(X-x \mid X>x)=\frac{1}{1-F(x)} \int_{x}^{\infty}[1-F(t)] d t
$$

Bayramoglu and Gurler [13] study the mean residual life function of $k$ out of $n$ system with nonidentical components while Govil and Aggarwal [14] and Abdous and Berred [15] compared for different distributions like gamma, exponential, Pareto, uniform, truncated normal, Maxwell, 
TABLE 9: Bayes estimator and posterior risk of stress and strength parameter under SELF.

\begin{tabular}{|c|c|c|c|c|c|c|}
\hline \multirow{2}{*}{$\begin{array}{l}n \\
R\end{array}$} & \multicolumn{3}{|c|}{ UP } & \multicolumn{3}{|c|}{$\mathrm{JP}$} \\
\hline & 0.026364 & 0.0604 & 0.998771 & 0.026364 & 0.0604 & 0.998771 \\
\hline 20,40 & $\begin{array}{c}0.028511 \\
(0.000077)\end{array}$ & $\begin{array}{c}0.064361 \\
(0.000288)\end{array}$ & $\begin{array}{c}0.998763 \\
\left(1.44921 * 10^{-7}\right)\end{array}$ & $\begin{array}{c}0.027985 \\
(0.000078)\end{array}$ & $\begin{array}{c}0.063217 \\
(0.000282)\end{array}$ & $\begin{array}{c}0.998728 \\
\left(1.40568 * 10^{-7}\right)\end{array}$ \\
\hline 60,80 & $\begin{array}{c}0.027029 \\
(0.000030)\end{array}$ & $\begin{array}{c}0.061592 \\
(0.000109)\end{array}$ & $\begin{array}{c}0.998756 \\
\left(6.22876 * 10^{-8}\right)\end{array}$ & $\begin{array}{c}0.026962 \\
(0.000030)\end{array}$ & $\begin{array}{c}0.061386 \\
(0.000109)\end{array}$ & $\begin{array}{c}0.998749 \\
\left(6.19239 * 10^{-8}\right)\end{array}$ \\
\hline 80,100 & $\begin{array}{c}0.026832 \\
(0.000023)\end{array}$ & $\begin{array}{c}0.061133 \\
(0.000084)\end{array}$ & $\begin{array}{c}0.998754 \\
\left(4.89937 * 10^{-8}\right)\end{array}$ & $\begin{array}{c}0.026797 \\
(0.000023)\end{array}$ & $\begin{array}{c}0.061068 \\
(0.000084)\end{array}$ & $\begin{array}{c}0.998749 \\
\left(4.88093 * 10^{-8}\right)\end{array}$ \\
\hline 80,60 & $\begin{array}{c}0.026663 \\
(0.000029)\end{array}$ & $\begin{array}{c}0.060702 \\
(0.000105)\end{array}$ & $\begin{array}{c}0.998735 \\
\left(6.74031 * 10^{-8}\right)\end{array}$ & $\begin{array}{c}0.026796 \\
(0.000029)\end{array}$ & $\begin{array}{c}0.061019 \\
(0.000105)\end{array}$ & $\begin{array}{c}0.998738 \\
\left(6.75851 * 10^{-8}\right)\end{array}$ \\
\hline 100,60 & $\begin{array}{c}0.026575 \\
(0.000026)\end{array}$ & $\begin{array}{c}0.060422 \\
(0.000095)\end{array}$ & $\begin{array}{c}0.998733 \\
\left(6.31669 * 10^{-8}\right)\end{array}$ & $\begin{array}{c}0.026766 \\
(0.000026)\end{array}$ & $\begin{array}{c}0.060865 \\
(0.000095)\end{array}$ & $\begin{array}{c}0.99874 \\
\left(6.31061 * 10^{-8}\right)\end{array}$ \\
\hline$n$ & & LMP & & & GP & \\
\hline$R$ & 0.026364 & 0.0604 & 0.998771 & 0.026364 & 0.0604 & 0.998771 \\
\hline 20,40 & $\begin{array}{c}0.028581 \\
(0.000076)\end{array}$ & $\begin{array}{c}0.064860 \\
(0.000281)\end{array}$ & $\begin{array}{c}0.99874 \\
\left(1.40336 * 10^{-7}\right)\end{array}$ & $\begin{array}{c}0.027987 \\
(0.000078)\end{array}$ & $\begin{array}{c}0.063762 \\
(0.000280)\end{array}$ & $\begin{array}{c}0.99871 \\
\left(1.37326 * 10^{-7}\right)\end{array}$ \\
\hline 60,80 & $\begin{array}{c}0.027051 \\
(0.000029)\end{array}$ & $\begin{array}{c}0.061760 \\
(0.000109)\end{array}$ & $\begin{array}{c}0.998748 \\
\left(6.18727 * 10^{-8}\right)\end{array}$ & $\begin{array}{c}0.026956 \\
(0.000029)\end{array}$ & $\begin{array}{c}0.061625 \\
(0.000108)\end{array}$ & $\begin{array}{c}0.998743 \\
\left(6.1525 * 10^{-8}\right)\end{array}$ \\
\hline 80,100 & $\begin{array}{c}0.026848 \\
(0.000022)\end{array}$ & $\begin{array}{c}0.061323 \\
(0.000083)\end{array}$ & $\begin{array}{c}0.998748 \\
\left(4.87444 * 10^{-8}\right)\end{array}$ & $\begin{array}{c}0.026792 \\
(0.000022)\end{array}$ & $\begin{array}{c}0.061255 \\
(0.000083)\end{array}$ & $\begin{array}{c}0.998745 \\
\left(4.85798 * 10^{-8}\right)\end{array}$ \\
\hline 80,60 & $\begin{array}{c}0.026676 \\
(0.000028)\end{array}$ & $\begin{array}{c}0.061014 \\
(0.000105)\end{array}$ & $\begin{array}{c}0.998729 \\
\left(6.69801 * 10^{-8}\right)\end{array}$ & $\begin{array}{c}0.026781 \\
(0.000028)\end{array}$ & $\begin{array}{c}0.061306 \\
(0.000104)\end{array}$ & $\begin{array}{c}0.998733 \\
\left(6.73044 * 10^{-8}\right)\end{array}$ \\
\hline 100,60 & $\begin{array}{c}0.026585 \\
(0.000026)\end{array}$ & $\begin{array}{c}0.060732 \\
(0.000094)\end{array}$ & $\begin{array}{c}0.998728 \\
\left(6.28193 * 10^{-8}\right)\end{array}$ & $\begin{array}{c}0.026748 \\
(0.000026)\end{array}$ & $\begin{array}{c}0.061143 \\
(0.000094)\end{array}$ & $\begin{array}{c}0.998736 \\
\left(6.23054 * 10^{-8}\right)\end{array}$ \\
\hline
\end{tabular}

TABLE 10: Bayes estimator and posterior risk of stress and strength parameter under WSELF.

\begin{tabular}{|c|c|c|c|c|c|c|}
\hline \multirow{2}{*}{$\begin{array}{l}n \\
R\end{array}$} & \multicolumn{3}{|c|}{ UP } & \multicolumn{3}{|c|}{ JP } \\
\hline & 0.026364 & 0.0604 & 0.998771 & 0.026364 & 0.0604 & 0.998771 \\
\hline 20,40 & $\begin{array}{c}0.025616 \\
(0.002896)\end{array}$ & $\begin{array}{c}0.059646 \\
(0.004714)\end{array}$ & $\begin{array}{c}0.998762 \\
\left(1.4508 * 10^{-7}\right)\end{array}$ & $\begin{array}{c}0.025146 \\
(0.002839)\end{array}$ & $\begin{array}{c}0.058595 \\
(0.004622)\end{array}$ & $\begin{array}{c}0.998728 \\
\left(1.40713 * 10^{-7}\right)\end{array}$ \\
\hline 60,80 & $\begin{array}{c}0.025913 \\
(0.001116)\end{array}$ & $\begin{array}{c}0.059791 \\
(0.001801)\end{array}$ & $\begin{array}{c}0.998756 \\
\left(6.2361 * 10^{-8}\right)\end{array}$ & $\begin{array}{l}0.025849 \\
(0.001113)\end{array}$ & $\begin{array}{c}0.059591 \\
(0.001795)\end{array}$ & $\begin{array}{c}0.998749 \\
\left(6.1996 * 10^{-8}\right)\end{array}$ \\
\hline 80,100 & $\begin{array}{c}0.025971 \\
(0.000860)\end{array}$ & $\begin{array}{c}0.059748 \\
(0.001384)\end{array}$ & $\begin{array}{c}0.998754 \\
\left(4.90522 * 10^{-8}\right)\end{array}$ & $\begin{array}{c}0.025938 \\
(0.000859)\end{array}$ & $\begin{array}{c}0.059685 \\
(0.001383)\end{array}$ & $\begin{array}{c}0.998749 \\
\left(4.88671 * 10^{-8}\right)\end{array}$ \\
\hline 80,60 & $\begin{array}{c}0.025571 \\
(0.001092)\end{array}$ & $\begin{array}{c}0.058960 \\
(0.001742)\end{array}$ & $\begin{array}{c}0.998735 \\
\left(6.74825 * 10^{-8}\right)\end{array}$ & $\begin{array}{c}0.025699 \\
(0.001091)\end{array}$ & $\begin{array}{c}0.059267 \\
(0.001742)\end{array}$ & $\begin{array}{c}0.998738 \\
\left(6.74652 * 10^{-8}\right)\end{array}$ \\
\hline 100,60 & $\begin{array}{c}0.025581 \\
(0.000993)\end{array}$ & $\begin{array}{c}0.058846 \\
(0.001576)\end{array}$ & $\begin{array}{c}0.998733 \\
\left(6.32415 * 10^{-8}\right)\end{array}$ & $\begin{array}{c}0.025765 \\
(0.009991)\end{array}$ & $\begin{array}{c}0.059276 \\
(0.001568)\end{array}$ & $\begin{array}{c}0.99874 \\
\left(6.31859 * 10^{-8}\right)\end{array}$ \\
\hline$n$ & & $\mathrm{CP}$ & & & GP & \\
\hline$R$ & 0.026364 & 0.0604 & 0.998771 & 0.026364 & 0.0604 & 0.998771 \\
\hline 20,40 & $\begin{array}{c}0.025679 \\
(0.002801)\end{array}$ & $\begin{array}{c}0.060117 \\
(0.004543)\end{array}$ & $\begin{array}{c}0.99874 \\
\left(1.40486 * 10^{-7}\right)\end{array}$ & $\begin{array}{c}0.025148 \\
(0.002839)\end{array}$ & $\begin{array}{c}0.059091 \\
(0.004571)\end{array}$ & $\begin{array}{c}0.99871 \\
\left(1.37462 * 10^{-7}\right)\end{array}$ \\
\hline 60,80 & $\begin{array}{l}0.025934 \\
(0.001111)\end{array}$ & $\begin{array}{c}0.059954 \\
(0.001786)\end{array}$ & $\begin{array}{c}0.998748 \\
\left(6.19446 * 10^{-8}\right)\end{array}$ & $\begin{array}{c}0.025843 \\
(0.001110)\end{array}$ & $\begin{array}{c}0.059822 \\
(0.001702)\end{array}$ & $\begin{array}{c}0.998743 \\
\left(6.15958 * 10^{-8}\right)\end{array}$ \\
\hline 80,100 & $\begin{array}{c}0.025987 \\
(0.000860)\end{array}$ & $\begin{array}{c}0.059934 \\
(0.001382)\end{array}$ & $\begin{array}{c}0.998748 \\
\left(4.8802 * 10^{-8}\right)\end{array}$ & $\begin{array}{c}0.025933 \\
(0.000859)\end{array}$ & $\begin{array}{c}0.059869 \\
(0.001382)\end{array}$ & $\begin{array}{c}0.998745 \\
\left(4.86369 * 10^{-8}\right)\end{array}$ \\
\hline 80,60 & $\begin{array}{c}0.025584 \\
(0.001092)\end{array}$ & $\begin{array}{c}0.059262 \\
(0.001741)\end{array}$ & $\begin{array}{c}0.998729 \\
\left(6.70581 * 10^{-8}\right)\end{array}$ & $\begin{array}{c}0.025684 \\
(0.001091)\end{array}$ & $\begin{array}{c}0.059548 \\
(0.001737)\end{array}$ & $\begin{array}{c}0.998733 \\
\left(6.70385 * 10^{-8}\right)\end{array}$ \\
\hline 100,60 & $\begin{array}{c}0.025591 \\
(0.000993)\end{array}$ & $\begin{array}{c}0.059146 \\
(0.001558)\end{array}$ & $\begin{array}{c}0.998728 \\
\left(6.28928 * 10^{-8}\right)\end{array}$ & $\begin{array}{c}0.025747 \\
(0.00990)\end{array}$ & $\begin{array}{c}0.059549 \\
(0.001553)\end{array}$ & $\begin{array}{c}0.998736 \\
\left(6.23805 * 10^{-8}\right)\end{array}$ \\
\hline
\end{tabular}


TABLE 11: Bayes estimator and posterior risk of stress and strength parameter under MSELF.

\begin{tabular}{|c|c|c|c|c|c|c|}
\hline$n$ & & UP & & & $\mathrm{JP}$ & \\
\hline$R$ & 0.026364 & 0.0604 & 0.998771 & 0.026364 & 0.0604 & 0.998771 \\
\hline 20,40 & $\begin{array}{c}0.023332 \\
(0.999986)\end{array}$ & $\begin{array}{c}0.055666 \\
(0.999815)\end{array}$ & $\begin{array}{c}0.998762 \\
(0.003708)\end{array}$ & $\begin{array}{c}0.023021 \\
(0.999987)\end{array}$ & $\begin{array}{c}0.054887 \\
(0.999813)\end{array}$ & $\begin{array}{c}0.998728 \\
(0.003812)\end{array}$ \\
\hline 60,80 & $\begin{array}{c}0.024906 \\
(0.999984)\end{array}$ & $\begin{array}{c}0.058118 \\
(0.999798)\end{array}$ & $\begin{array}{c}0.998756 \\
(0.003728)\end{array}$ & $\begin{array}{c}0.024851 \\
(0.999984)\end{array}$ & $\begin{array}{c}0.057935 \\
(0.999800)\end{array}$ & $\begin{array}{c}0.998749 \\
(0.003729)\end{array}$ \\
\hline 80,100 & $\begin{array}{c}0.025178 \\
(0.999984)\end{array}$ & $\begin{array}{c}0.058442 \\
(0.999796)\end{array}$ & $\begin{array}{c}0.998754 \\
(0.003734)\end{array}$ & $\begin{array}{c}0.025149 \\
(0.999984)\end{array}$ & $\begin{array}{c}0.058385 \\
(0.999797)\end{array}$ & $\begin{array}{c}0.998749 \\
(0.003734)\end{array}$ \\
\hline 80,60 & $\begin{array}{c}0.024596 \\
(0.999985)\end{array}$ & $\begin{array}{c}0.057357 \\
(0.999806)\end{array}$ & $\begin{array}{c}0.998735 \\
(0.003790)\end{array}$ & $\begin{array}{c}0.024705 \\
(0.999984)\end{array}$ & $\begin{array}{c}0.057630 \\
(0.999803)\end{array}$ & $\begin{array}{c}0.998738 \\
(0.003780)\end{array}$ \\
\hline 100,60 & $\begin{array}{c}0.024689 \\
(0.999984)\end{array}$ & $\begin{array}{c}0.057390 \\
(0.999806)\end{array}$ & $\begin{array}{c}0.998733 \\
(0.003796)\end{array}$ & $\begin{array}{c}0.024846 \\
(0.999984)\end{array}$ & $\begin{array}{c}0.0577777 \\
(0.999802)\end{array}$ & $\begin{array}{c}0.99874 \\
(0.003776)\end{array}$ \\
\hline$n$ & & LMP & & & GP & \\
\hline$R$ & 0.026364 & 0.0604 & 0.998771 & 0.026364 & 0.0604 & 0.998771 \\
\hline 20,40 & $\begin{array}{c}0.023375 \\
(0.999986)\end{array}$ & $\begin{array}{c}0.056022 \\
(0.999811)\end{array}$ & $\begin{array}{c}0.99874 \\
(0.003706)\end{array}$ & $\begin{array}{c}0.023023 \\
(0.999987)\end{array}$ & $\begin{array}{c}0.055252 \\
(0.999802)\end{array}$ & $\begin{array}{c}0.998709 \\
(0.003666)\end{array}$ \\
\hline 60,80 & $\begin{array}{c}0.024924 \\
(0.999984)\end{array}$ & $\begin{array}{c}0.058258 \\
(0.999797)\end{array}$ & $\begin{array}{c}0.998748 \\
(0.003721)\end{array}$ & $\begin{array}{c}0.024847 \\
(0.999984)\end{array}$ & $\begin{array}{c}0.058141 \\
(0.999796)\end{array}$ & $\begin{array}{c}0.998743 \\
(0.003727)\end{array}$ \\
\hline 80,100 & $\begin{array}{c}0.025191 \\
(0.999984)\end{array}$ & $\begin{array}{c}0.058613 \\
(0.999794)\end{array}$ & $\begin{array}{c}0.998748 \\
(0.003732)\end{array}$ & $\begin{array}{c}0.025144 \\
(0.999984)\end{array}$ & $\begin{array}{c}0.058552 \\
(0.999795)\end{array}$ & $\begin{array}{c}0.998745 \\
(0.003731)\end{array}$ \\
\hline 80,60 & $\begin{array}{c}0.024607 \\
(0.999985)\end{array}$ & $\begin{array}{c}0.057626 \\
(0.999803)\end{array}$ & $\begin{array}{c}0.998728 \\
(0.003780)\end{array}$ & $\begin{array}{c}0.024692 \\
(0.999984)\end{array}$ & $\begin{array}{c}0.057881 \\
(0.99980)\end{array}$ & $\begin{array}{c}0.998733 \\
(0.003759)\end{array}$ \\
\hline 100,60 & $\begin{array}{c}0.024697 \\
(0.999984)\end{array}$ & $\begin{array}{c}0.057659 \\
(0.999803)\end{array}$ & $\begin{array}{c}0.998728 \\
(0.003712)\end{array}$ & $\begin{array}{c}0.024831 \\
(0.999984)\end{array}$ & $\begin{array}{c}0.058025 \\
(0.99980)\end{array}$ & $\begin{array}{c}0.998736 \\
(0.003708)\end{array}$ \\
\hline
\end{tabular}

TABLE 12: Bayes estimator and posterior risk of stress and strength parameter under PLF.

\begin{tabular}{|c|c|c|c|c|c|c|}
\hline \multirow{2}{*}{$\begin{array}{l}n \\
R\end{array}$} & \multicolumn{3}{|c|}{ UP } & \multicolumn{3}{|c|}{ JP } \\
\hline & 0.026364 & 0.0604 & 0.998771 & 0.026364 & 0.0604 & 0.998771 \\
\hline 20,40 & $\begin{array}{c}0.029827 \\
(0.002631)\end{array}$ & $\begin{array}{c}0.066557 \\
(0.004393)\end{array}$ & $\begin{array}{c}0.998763 \\
\left(1.451 * 10^{-7}\right)\end{array}$ & $\begin{array}{c}0.029348 \\
(0.002628)\end{array}$ & $\begin{array}{c}0.065490 \\
(0.004345)\end{array}$ & $\begin{array}{c}0.998728 \\
\left(1.4747 * 10^{-7}\right)\end{array}$ \\
\hline 60,80 & $\begin{array}{c}0.027570 \\
(0.001082)\end{array}$ & $\begin{array}{c}0.062474 \\
(0.001764)\end{array}$ & $\begin{array}{c}0.998756 \\
\left(6.23652 * 10^{-8}\right)\end{array}$ & $\begin{array}{c}0.027506 \\
(0.001087)\end{array}$ & $\begin{array}{c}0.062271 \\
(0.001769)\end{array}$ & $\begin{array}{c}0.998749 \\
\left(6.24015 * 10^{-8}\right)\end{array}$ \\
\hline 80,100 & $\begin{array}{c}0.027252 \\
(0.000841)\end{array}$ & $\begin{array}{c}0.061814 \\
(0.001363)\end{array}$ & $\begin{array}{c}0.998754 \\
\left(4.90548 * 10^{-8}\right)\end{array}$ & $\begin{array}{c}0.027219 \\
(0.000843)\end{array}$ & $\begin{array}{c}0.061751 \\
(0.001361)\end{array}$ & $\begin{array}{c}0.998749 \\
\left(4.88704 * 10^{-8}\right)\end{array}$ \\
\hline 80,60 & $\begin{array}{c}0.027198 \\
(0.001070)\end{array}$ & $\begin{array}{c}0.061565 \\
(0.001725)\end{array}$ & $\begin{array}{c}0.998735 \\
\left(6.74885 * 10^{-8}\right)\end{array}$ & $\begin{array}{c}0.027326 \\
(0.001059)\end{array}$ & $\begin{array}{c}0.061873 \\
(0.001707)\end{array}$ & $\begin{array}{c}0.998738 \\
\left(6.75706 * 10^{-8}\right)\end{array}$ \\
\hline 100,60 & $\begin{array}{c}0.027064 \\
(0.000979)\end{array}$ & $\begin{array}{c}0.061206 \\
(0.001568)\end{array}$ & $\begin{array}{c}0.998733 \\
\left(6.3247 * 10^{-8}\right)\end{array}$ & $\begin{array}{c}0.027248 \\
(0.000966)\end{array}$ & $\begin{array}{c}0.061638 \\
(0.001546)\end{array}$ & $\begin{array}{c}0.99874 \\
\left(6.32386 * 10^{-8}\right)\end{array}$ \\
\hline$n$ & & LMP & & & GP & \\
\hline$R$ & 0.026364 & 0.0604 & 0.998771 & 0.026364 & 0.0604 & 0.998771 \\
\hline 20,40 & $\begin{array}{l}0.0298893 \\
(0.002617)\end{array}$ & $\begin{array}{c}0.067019 \\
(0.004317)\end{array}$ & $\begin{array}{c}0.99874 \\
\left(1.42516 * 10^{-7}\right)\end{array}$ & $\begin{array}{c}0.029351 \\
(0.002627)\end{array}$ & $\begin{array}{c}0.066001 \\
(0.004417)\end{array}$ & $\begin{array}{c}0.99871 \\
\left(1.37503 * 10^{-7}\right)\end{array}$ \\
\hline 60,80 & $\begin{array}{c}0.027591 \\
(0.001080)\end{array}$ & $\begin{array}{c}0.062633 \\
(0.001748)\end{array}$ & $\begin{array}{c}0.998748 \\
\left(6.19503 * 10^{-8}\right)\end{array}$ & $\begin{array}{c}0.027500 \\
(0.001080)\end{array}$ & $\begin{array}{c}0.062503 \\
(0.001746)\end{array}$ & $\begin{array}{c}0.998743 \\
\left(6.16024 * 10^{-8}\right)\end{array}$ \\
\hline 80,100 & $\begin{array}{c}0.027268 \\
(0.000839)\end{array}$ & $\begin{array}{c}0.061999 \\
(0.001354)\end{array}$ & $\begin{array}{c}0.998748 \\
\left(4.88056 * 10^{-8}\right)\end{array}$ & $\begin{array}{c}0.027214 \\
(0.000834)\end{array}$ & $\begin{array}{c}0.061934 \\
(0.001347)\end{array}$ & $\begin{array}{c}0.998745 \\
\left(4.86409 * 10^{-8}\right)\end{array}$ \\
\hline 80,60 & $\begin{array}{c}0.027211 \\
(0.001058)\end{array}$ & $\begin{array}{c}0.061868 \\
(0.001707)\end{array}$ & $\begin{array}{c}0.998729 \\
\left(6.70654 * 10^{-8}\right)\end{array}$ & $\begin{array}{c}0.027311 \\
(0.001060)\end{array}$ & $\begin{array}{c}0.062150 \\
(0.001689)\end{array}$ & $\begin{array}{c}0.998733 \\
\left(6.69898 * 10^{-8}\right)\end{array}$ \\
\hline 100,60 & $\begin{array}{c}0.027074 \\
(0.000958)\end{array}$ & $\begin{array}{c}0.061508 \\
(0.001543)\end{array}$ & $\begin{array}{c}0.998728 \\
\left(6.28994 * 10^{-8}\right)\end{array}$ & $\begin{array}{c}0.027231 \\
(0.000957)\end{array}$ & $\begin{array}{c}0.061908 \\
(0.001529)\end{array}$ & $\begin{array}{c}0.998736 \\
\left(6.23856 * 10^{-8}\right)\end{array}$ \\
\hline
\end{tabular}


TABLE 13: Bayes estimator and posterior risk of stress and strength parameter under SLLF.

\begin{tabular}{|c|c|c|c|c|c|c|}
\hline \multirow{2}{*}{$\begin{array}{l}n \\
R\end{array}$} & \multicolumn{3}{|c|}{ UP } & \multicolumn{3}{|c|}{$\mathrm{JP}$} \\
\hline & 0.026364 & 0.0604 & 0.998771 & 0.026364 & 0.0604 & 0.998771 \\
\hline 20,40 & $\begin{array}{l}0.027033 \\
(0.11001)\end{array}$ & $\begin{array}{c}0.061970 \\
(0.077534)\end{array}$ & $\begin{array}{c}0.998762 \\
\left(1.4526 * 10^{-7}\right)\end{array}$ & $\begin{array}{c}0.026509 \\
(0.109867)\end{array}$ & $\begin{array}{c}0.060832 \\
(0.077376)\end{array}$ & $\begin{array}{c}0.998728 \\
\left(1.40892 * 10^{-7}\right)\end{array}$ \\
\hline 60,80 & $\begin{array}{c}0.026465 \\
(0.042628)\end{array}$ & $\begin{array}{c}0.060684 \\
(0.029901)\end{array}$ & $\begin{array}{c}0.998756 \\
\left(6.24387 * 10^{-8}\right)\end{array}$ & $\begin{array}{c}0.026398 \\
(0.042621)\end{array}$ & $\begin{array}{c}0.060480 \\
(0.029899)\end{array}$ & $\begin{array}{c}0.998749 \\
\left(6.20736 * 10^{-8}\right)\end{array}$ \\
\hline 80,100 & $\begin{array}{c}0.026398 \\
(0.032862)\end{array}$ & $\begin{array}{c}0.060436 \\
(0.023035)\end{array}$ & $\begin{array}{c}0.998754 \\
\left(4.91134 * 10^{-8}\right)\end{array}$ & $\begin{array}{c}0.026363 \\
(0.032859)\end{array}$ & $\begin{array}{c}0.060372 \\
(0.023033)\end{array}$ & $\begin{array}{c}0.998749 \\
\left(4.89283 * 10^{-8}\right)\end{array}$ \\
\hline 80,60 & $\begin{array}{c}0.026109 \\
(0.042241)\end{array}$ & $\begin{array}{c}0.059822 \\
(0.029329)\end{array}$ & $\begin{array}{c}0.998735 \\
\left(6.7568 * 10^{-8}\right)\end{array}$ & $\begin{array}{c}0.026243 \\
(0.042241)\end{array}$ & $\begin{array}{c}0.060138 \\
(0.029323)\end{array}$ & $\begin{array}{c}0.998738 \\
\left(6.75607 * 10^{-8}\right)\end{array}$ \\
\hline 100,60 & $\begin{array}{c}0.026071 \\
(0.038466) \\
\end{array}$ & $\begin{array}{c}0.059625 \\
(0.026609) \\
\end{array}$ & $\begin{array}{c}0.998733 \\
\left(6.33217 * 10^{-8}\right)\end{array}$ & $\begin{array}{c}0.026262 \\
(0.038458) \\
\end{array}$ & $\begin{array}{c}0.060068 \\
(0.026605) \\
\end{array}$ & $\begin{array}{c}0.99874 \\
\left(6.32621 * 10^{-8}\right) \\
\end{array}$ \\
\hline$n$ & & LMP & & & GP & \\
\hline$R$ & 0.026364 & 0.0604 & 0.998771 & 0.026364 & 0.0604 & 0.998771 \\
\hline 20,40 & $\begin{array}{c}0.027104 \\
(0.109771)\end{array}$ & $\begin{array}{c}0.062474 \\
(0.077367)\end{array}$ & $\begin{array}{c}0.99874 \\
\left(1.40666 * 10^{-7}\right)\end{array}$ & $\begin{array}{c}0.026511 \\
(0.109817)\end{array}$ & $\begin{array}{c}0.061372 \\
(0.077337)\end{array}$ & $\begin{array}{c}0.99871 \\
\left(1.37639 * 10^{-7}\right)\end{array}$ \\
\hline 60,80 & $\begin{array}{c}0.026487 \\
(0.042620)\end{array}$ & $\begin{array}{c}0.060853 \\
(0.029893)\end{array}$ & $\begin{array}{c}0.998748 \\
\left(6.20223 * 10^{-8}\right)\end{array}$ & $\begin{array}{c}0.026392 \\
(0.042619)\end{array}$ & $\begin{array}{c}0.060718 \\
(0.029804)\end{array}$ & $\begin{array}{c}0.998743 \\
\left(6.16733 * 10^{-8}\right)\end{array}$ \\
\hline 80,100 & $\begin{array}{c}0.026414 \\
(0.032860)\end{array}$ & $\begin{array}{c}0.060626 \\
(0.023029)\end{array}$ & $\begin{array}{c}0.998748 \\
\left(4.88632 * 10^{-8}\right)\end{array}$ & $\begin{array}{c}0.026358 \\
(0.032858)\end{array}$ & $\begin{array}{c}0.060559 \\
(0.023030)\end{array}$ & $\begin{array}{c}0.998745 \\
\left(4.8698 * 10^{-8}\right)\end{array}$ \\
\hline 80,60 & $\begin{array}{c}0.026123 \\
(0.042240)\end{array}$ & $\begin{array}{c}0.060133 \\
(0.029322)\end{array}$ & $\begin{array}{c}0.998729 \\
\left(6.71435 * 10^{-8}\right)\end{array}$ & $\begin{array}{c}0.026227 \\
(0.042235)\end{array}$ & $\begin{array}{c}0.060425 \\
(0.029307)\end{array}$ & $\begin{array}{c}0.998733 \\
\left(6.7069 * 10^{-8}\right)\end{array}$ \\
\hline 100,60 & $\begin{array}{c}0.026081 \\
(0.038459)\end{array}$ & $\begin{array}{c}0.059934 \\
(0.026602)\end{array}$ & $\begin{array}{c}0.998728 \\
\left(6.29729 * 10^{-8}\right)\end{array}$ & $\begin{array}{c}0.026244 \\
(0.038455)\end{array}$ & $\begin{array}{c}0.060347 \\
(0.026580)\end{array}$ & $\begin{array}{c}0.998736 \\
\left(6.24607 * 10^{-8}\right)\end{array}$ \\
\hline
\end{tabular}

and lognormal distributions. The mean residual life function for Lindley distribution is

$$
m(x)=\frac{\theta+2+\theta x}{\theta(\theta+1+\theta x)}
$$

Ghitany et al. $[2,5]$ point out the following remarks.

$$
m(0)=\mu
$$

$m(x)$ is a decreasing function in $x$ and $\theta$ and $1 / \theta<$ $m(x)<(\theta+2) /(\theta(\theta+1))=\mu$.

From $m(x)$, one can easily observe that mean residuals life is a diminishing function of time because the distribution belongs to exponential family and for larger parameter value it is close to zero. To evaluate the Bayes estimates and their risk, since the integral appears in both numerator and denumerator, we required a suitable approximate method to obtain Bayes estimates and respective posterior risks. The simplest method is Lindley's [16] approximation method, which approaches the ratio of the integrals as a whole and produces a single numerical results. Thus, Lindley approximation (LA) given by Lindley [16] for obtaining the Bayes estimator and posterior risk of $\theta$ (Mathematica can be used for the solution of integral but takes large time as compared to LA). Many researchers have used this approximation for solving the ratio of integrals for different numbers of parameters for lifetime distributions; see among others Howlader and Hossain [17], Singh et al. [18], and Preda et al. [19].
If $n$ is sufficiently large, the ratio of the integral of the form according to Lindley [16] can be computed as

$$
I(x)=E[u(\theta)]=\frac{\int_{\theta} u(\theta) \exp [l(\theta, \mathbf{x})+g(\theta)] d \theta}{\int_{\theta} \exp [l(\theta, \mathbf{x})+g(\theta)] d \theta}, \quad \theta>0,
$$

where $u(\theta)=$ function of $\theta$ only; $l(\theta, \mathbf{x})=\log$ of likelihood; $g(\theta)=\log$ of prior of $\theta$, can be evaluated as

$$
\begin{aligned}
I(x)= & u(\widehat{\theta})+0.5\left[\left(\hat{u}_{\theta \theta}+2 \hat{u}_{\theta} \bar{p}_{\theta}\right) \hat{\sigma}_{\theta \theta}\right] \\
& +0.5\left[\left(\hat{u}_{\theta} \hat{\sigma}_{\theta \theta}\right)\left(\hat{L}_{\theta \theta \theta} \hat{\sigma}_{\theta \theta}\right)\right]
\end{aligned}
$$

where $\hat{\theta}=M L E$ of $\theta=\left(-(\bar{x}-1)+\sqrt{(\bar{x}-1)^{2}+8 \bar{x}}\right) /$ $2 \bar{x}, \bar{x}>0 ; \bar{u}_{\theta}=\partial u(\widehat{\theta}) / \partial \widehat{\theta} ; \bar{u}_{\theta \theta}=\partial^{2} u(\widehat{\theta}) / \partial \widehat{\theta}^{2} ; \hat{L}_{\theta \theta \theta}=\partial^{3} l(\widehat{\theta}) /$ $\partial \hat{\theta}^{3} ; \bar{p}_{\theta}=\partial g(\widehat{\theta}) / \partial \widehat{\theta} ; \bar{\sigma}_{\theta \theta}=-1 / \hat{L}_{\theta \theta} ; \hat{L}_{\theta \theta}=\partial^{2} l(\widehat{\theta}) / \partial \widehat{\theta}^{2} ;$ the simulation study of mean residual life function (MRLF) for different loss functions under different prior is using $t=3$ (see Tables 2, 3, 4, 5, 6, 7, and 8).

Using SELF for $\theta=7.142857$, Bayes estimates are overestimated while for $\theta=1.2$ and 0.114114 results are underestimated and by increasing sample size these approaches to true parameter values. This behaviour can be observed in all loss functions except PLF. Making comparison between symmetric and asymmetric loss functions, one can 
TABLE 14: Bayes estimator and posterior risk of stress and strength parameter under ELF.

\begin{tabular}{|c|c|c|c|c|c|c|}
\hline$n$ & \multicolumn{3}{|c|}{ UP } & \multicolumn{3}{|c|}{$\mathrm{JP}$} \\
\hline$R$ & 0.026364 & 0.0604 & 0.998771 & 0.026364 & 0.0604 & 0.998771 \\
\hline 20,40 & $\begin{array}{l}0.025616 \\
(7.27524)\end{array}$ & $\begin{array}{l}0.059646 \\
(5.60043)\end{array}$ & $\begin{array}{c}0.998762 \\
(0.002477)\end{array}$ & $\begin{array}{l}0.025146 \\
(7.21335)\end{array}$ & $\begin{array}{l}0.058595 \\
(5.53674)\end{array}$ & $\begin{array}{c}0.998728 \\
(0.002446)\end{array}$ \\
\hline 60,80 & $\begin{array}{c}0.025913 \\
(7.28493)\end{array}$ & $\begin{array}{c}0.059791 \\
(5.61898)\end{array}$ & $\begin{array}{c}0.998756 \\
(0.002490)\end{array}$ & $\begin{array}{l}0.025849 \\
(7.27996)\end{array}$ & $\begin{array}{c}0.059591 \\
(5.56257)\end{array}$ & $\begin{array}{c}0.998749 \\
(0.002450)\end{array}$ \\
\hline 80,100 & $\begin{array}{l}0.025971 \\
(7.28523)\end{array}$ & $\begin{array}{l}0.059748 \\
(5.62379)\end{array}$ & $\begin{array}{c}0.998754 \\
(0.002494)\end{array}$ & $\begin{array}{l}0.025938 \\
(7.28483)\end{array}$ & $\begin{array}{l}0.059685 \\
(5.60591)\end{array}$ & $\begin{array}{c}0.998749 \\
(0.002443)\end{array}$ \\
\hline 80,60 & $\begin{array}{l}0.025571 \\
(7.31176)\end{array}$ & $\begin{array}{l}0.058960 \\
(5.64728)\end{array}$ & $\begin{array}{c}0.998735 \\
(0.002532)\end{array}$ & $\begin{array}{l}0.025699 \\
(7.30167)\end{array}$ & $\begin{array}{l}0.059267 \\
(5.62381)\end{array}$ & $\begin{array}{c}0.998738 \\
(0.002535)\end{array}$ \\
\hline 100,60 & $\begin{array}{l}0.025581 \\
(7.31282)\end{array}$ & $\begin{array}{l}0.058846 \\
(5.65251)\end{array}$ & $\begin{array}{c}0.998733 \\
(0.002535)\end{array}$ & $\begin{array}{l}0.025765 \\
(7.30984)\end{array}$ & $\begin{array}{l}0.059276 \\
(5.63837)\end{array}$ & $\begin{array}{c}0.99874 \\
(0.002522)\end{array}$ \\
\hline$n$ & \multicolumn{3}{|c|}{ LMP } & \multicolumn{3}{|c|}{ GP } \\
\hline$R$ & 0.026364 & 0.0604 & 0.998771 & 0.026364 & 0.0604 & 0.998771 \\
\hline 20,40 & $\begin{array}{l}0.025679 \\
(7.21076)\end{array}$ & $\begin{array}{c}0.060117 \\
(5.48446)\end{array}$ & $\begin{array}{c}0.99874 \\
(0.002452)\end{array}$ & $\begin{array}{l}0.025148 \\
(7.21315)\end{array}$ & $\begin{array}{l}0.059091 \\
(5.47148)\end{array}$ & $\begin{array}{c}0.99871 \\
(0.002452)\end{array}$ \\
\hline 60,80 & $\begin{array}{l}0.025934 \\
(7.23392)\end{array}$ & $\begin{array}{l}0.059954 \\
(5.53147)\end{array}$ & $\begin{array}{c}0.998748 \\
(0.002405)\end{array}$ & $\begin{array}{l}0.025843 \\
(7.23390)\end{array}$ & $\begin{array}{l}0.059822 \\
(5.53139)\end{array}$ & $\begin{array}{c}0.998743 \\
(0.002406)\end{array}$ \\
\hline 80,100 & $\begin{array}{l}0.025987 \\
(7.28404)\end{array}$ & $\begin{array}{l}0.059934 \\
(5.60153)\end{array}$ & $\begin{array}{c}0.998748 \\
(0.002366)\end{array}$ & $\begin{array}{l}0.025933 \\
(7.28403)\end{array}$ & $\begin{array}{l}0.059868 \\
(5.60174)\end{array}$ & $\begin{array}{c}0.998745 \\
(0.002312)\end{array}$ \\
\hline 80,60 & $\begin{array}{l}0.025584 \\
(7.30072)\end{array}$ & $\begin{array}{l}0.059262 \\
(5.61983)\end{array}$ & $\begin{array}{c}0.998729 \\
(0.002345)\end{array}$ & $\begin{array}{l}0.025684 \\
(7.30076)\end{array}$ & $\begin{array}{l}0.059548 \\
(5.61233)\end{array}$ & $\begin{array}{c}0.998733 \\
(0.002353)\end{array}$ \\
\hline 100,60 & $\begin{array}{l}0.025591 \\
(7.30237)\end{array}$ & $\begin{array}{c}0.059146 \\
(5.63426)\end{array}$ & $\begin{array}{c}0.998728 \\
(0.002246)\end{array}$ & $\begin{array}{l}0.025747 \\
(7.30235)\end{array}$ & $\begin{array}{l}0.059549 \\
(5.63259)\end{array}$ & $\begin{array}{c}0.998736 \\
(0.002240)\end{array}$ \\
\hline
\end{tabular}

TABLE 15: Bayes estimator and posterior risk of stress and strength parameter under KLF.

\begin{tabular}{|c|c|c|c|c|c|c|}
\hline$n$ & & UP & & & JP & \\
\hline$R$ & 0.026364 & 0.0604 & 0.998771 & 0.026364 & 0.0604 & 0.998771 \\
\hline 20,40 & $\begin{array}{c}0.027025 \\
(0.226074)\end{array}$ & $\begin{array}{c}0.061958 \\
(0.158076)\end{array}$ & $\begin{array}{c}0.998762 \\
\left(2.9052 * 10^{-7}\right)\end{array}$ & $\begin{array}{c}0.026527 \\
(0.225789)\end{array}$ & $\begin{array}{l}0.060863 \\
(0.15776)\end{array}$ & $\begin{array}{c}0.998728 \\
\left(2.81784 * 10^{-7}\right)\end{array}$ \\
\hline 60,80 & $\begin{array}{c}0.026465 \\
(0.086164)\end{array}$ & $\begin{array}{c}0.060685 \\
(0.060249)\end{array}$ & $\begin{array}{c}0.998756 \\
\left(1.24877 * 10^{-7}\right)\end{array}$ & $\begin{array}{c}0.026399 \\
(0.086151)\end{array}$ & $\begin{array}{c}0.060482 \\
(0.060245)\end{array}$ & $\begin{array}{c}0.998749 \\
\left(1.24147 * 10^{-7}\right)\end{array}$ \\
\hline 80,100 & $\begin{array}{c}0.026398 \\
(0.066263)\end{array}$ & $\begin{array}{c}0.060436 \\
(0.046336)\end{array}$ & $\begin{array}{c}0.998754 \\
\left(9.82267 * 10^{-8}\right)\end{array}$ & $\begin{array}{c}0.026364 \\
(0.066258)\end{array}$ & $\begin{array}{c}0.060373 \\
(0.046331)\end{array}$ & $\begin{array}{c}0.998749 \\
\left(9.78566 * 10^{-8}\right)\end{array}$ \\
\hline 80,60 & $\begin{array}{c}0.026111 \\
(0.085374)\end{array}$ & $\begin{array}{c}0.059825 \\
(0.059089)\end{array}$ & $\begin{array}{c}0.998735 \\
\left(1.35136 * 10^{-7}\right)\end{array}$ & $\begin{array}{c}0.026242 \\
(0.085368)\end{array}$ & $\begin{array}{c}0.060137 \\
(0.059076)\end{array}$ & $\begin{array}{c}0.998738 \\
\left(1.35101 * 10^{-7}\right)\end{array}$ \\
\hline 100,60 & $\begin{array}{c}0.026073 \\
(0.077673)\end{array}$ & $\begin{array}{c}0.059629 \\
(0.053573)\end{array}$ & $\begin{array}{c}0.998733 \\
\left(1.26643 * 10^{-7}\right)\end{array}$ & $\begin{array}{c}0.026260 \\
(0.077674)\end{array}$ & $\begin{array}{c}0.060066 \\
(0.053566)\end{array}$ & $\begin{array}{c}0.99874 \\
\left(1.26324 * 10^{-7}\right)\end{array}$ \\
\hline$n$ & & LMP & & & GP & \\
\hline$R$ & 0.026364 & 0.0604 & 0.998771 & 0.026364 & 0.0604 & 0.998771 \\
\hline 20,40 & $\begin{array}{c}0.027091 \\
(0.225697)\end{array}$ & $\begin{array}{c}0.062444 \\
(0.157728)\end{array}$ & $\begin{array}{c}0.99874 \\
\left(2.81332 * 10^{-7}\right)\end{array}$ & $\begin{array}{c}0.026529 \\
(0.225695)\end{array}$ & $\begin{array}{c}0.061382 \\
(0.157082)\end{array}$ & $\begin{array}{c}0.99871 \\
\left(2.75278 * 10^{-7}\right)\end{array}$ \\
\hline 60,80 & $\begin{array}{c}0.026486 \\
(0.086152)\end{array}$ & $\begin{array}{c}0.06085 \\
(0.060234)\end{array}$ & $\begin{array}{c}0.998748 \\
\left(1.24045 * 10^{-7}\right)\end{array}$ & $\begin{array}{c}0.026393 \\
(0.086148)\end{array}$ & $\begin{array}{c}0.060717 \\
(0.060235)\end{array}$ & $\begin{array}{c}0.998743 \\
\left(1.23347 * 10^{-7}\right)\end{array}$ \\
\hline 80,100 & $\begin{array}{c}0.026414 \\
(0.066253)\end{array}$ & $\begin{array}{c}0.060625 \\
(0.046324)\end{array}$ & $\begin{array}{c}0.998748 \\
\left(9.77264 * 10^{-8}\right)\end{array}$ & $\begin{array}{c}0.026359 \\
(0.066247)\end{array}$ & $\begin{array}{c}0.060558 \\
(0.046322)\end{array}$ & $\begin{array}{c}0.998745 \\
\left(9.73961 * 10^{-8}\right)\end{array}$ \\
\hline 80,60 & $\begin{array}{c}0.026125 \\
(0.085360)\end{array}$ & $\begin{array}{c}0.060132 \\
(0.059016)\end{array}$ & $\begin{array}{c}0.998729 \\
\left(1.34287 * 10^{-7}\right)\end{array}$ & $\begin{array}{c}0.026227 \\
(0.085300)\end{array}$ & $\begin{array}{c}0.060420 \\
(0.059014)\end{array}$ & $\begin{array}{c}0.998733 \\
\left(1.34238 * 10^{-7}\right)\end{array}$ \\
\hline 100,60 & $\begin{array}{c}0.026083 \\
(0.077670)\end{array}$ & $\begin{array}{c}0.059934 \\
(0.053519)\end{array}$ & $\begin{array}{c}0.998728 \\
\left(1.25946 * 10^{-7}\right)\end{array}$ & $\begin{array}{c}0.026243 \\
(0.077659)\end{array}$ & $\begin{array}{c}0.060341 \\
(0.053515)\end{array}$ & $\begin{array}{c}0.998736 \\
\left(1.25921 * 10^{-7}\right)\end{array}$ \\
\hline
\end{tabular}


TABLE 16: Waiting time (in minutes) before customer service in Bank A.

\begin{tabular}{llllllllll}
\hline 0.8 & 2.9 & 4.3 & 5.0 & 6.7 & 8.2 & 9.7 & 11.9 & 14.1 & 19.9 \\
0.8 & 3.1 & 4.3 & 5.3 & 6.9 & 8.6 & 9.8 & 12.4 & 15.4 & 20.6 \\
1.3 & 3.2 & 4.4 & 5.5 & 7.1 & 8.6 & 10.7 & 12.5 & 15.4 & 21.3 \\
1.5 & 3.3 & 4.4 & 5.7 & 7.1 & 8.6 & 10.9 & 12.9 & 17.3 & 21.4 \\
1.8 & 3.5 & 4.6 & 5.7 & 7.1 & 8.8 & 11.0 & 13.0 & 17.3 & 21.9 \\
1.9 & 3.6 & 4.7 & 6.1 & 7.1 & 8.8 & 11.0 & 13.1 & 18.1 & 23.0 \\
1.9 & 4.0 & 4.7 & 6.2 & 7.4 & 8.9 & 11.1 & 13.3 & 18.2 & 27.0 \\
2.1 & 4.1 & 4.8 & 6.2 & 7.6 & 8.9 & 11.2 & 13.6 & 18.4 & 31.6 \\
2.6 & 4.2 & 4.9 & 6.2 & 7.7 & 9.5 & 11.2 & 13.7 & 18.9 & 33.1 \\
2.7 & 4.2 & 4.9 & 6.3 & 8.0 & 9.6 & 11.5 & 13.9 & 19.0 & 38.5
\end{tabular}

TABLE 17: Waiting time (in minutes) before customer service in Bank B.

\begin{tabular}{llllllllll}
\hline 0.1 & 1.2 & 2.3 & 2.9 & 3.5 & 5.3 & 6.8 & 8.0 & 8.5 & 13.2 \\
0.2 & 1.8 & 2.3 & 3.1 & 3.9 & 5.6 & 7.3 & 8.5 & 11.0 & 13.7 \\
0.3 & 1.9 & 2.5 & 3.1 & 4.0 & 5.6 & 7.5 & 8.7 & 12.1 & 14.5 \\
0.7 & 2.0 & 2.6 & 3.2 & 4.2 & 6.2 & 7.7 & 9.5 & 12.3 & 16.0 \\
0.9 & 2.2 & 2.7 & 3.4 & 4.5 & 6.3 & 7.7 & 10.7 & 12.8 & 16.5 \\
1.1 & 2.3 & 2.7 & 3.4 & 4.7 & 6.6 & 8.0 & 10.9 & 12.9 & 28.0 \\
\hline
\end{tabular}

TABLE 18: K-S distances and associated $P$ value and MLE.

\begin{tabular}{lccc}
\hline Data set & K-S test & $P$ value & MLE \\
\hline Bank A & 0.065 & 0.721 & 0.187 \\
Bank B & 0.083 & 0.826 & 0.280 \\
\hline
\end{tabular}

easily observes that SELF has smaller posterior risk. Since there is defect in symmetric loss function, that is, SELF assign equal weight to over and under estimation, so we have to look for an alternative choice. In case of asymmetric loss functions; WSELF, SLLF, and PLF can be alternative choices.

\section{Lorenz Curve (See Figure 2)}

For a positive random variable $X$, the Lorenz curve is defined by the graph of the ratio

$$
L(F(x))=\frac{E(X \mid X \leq x) F(x)}{E(X)}
$$

against $F(x)$ with the properties $L(p) \leq p, L(0)=0$, and $L(1)=1$ for $0 \leq p \leq 1$. If $X$ represents annual income, then, $L(p)$ is the proportion of total income that accrues to individuals having the $100 p \%$ lowest incomes; see Gail and Gastwirth [20] for details of Lorenz curves. For the exponential distribution, it is well known that Lorenz curve is given by

$$
L(p)=p[p+(1-p) \log (1-p)]
$$
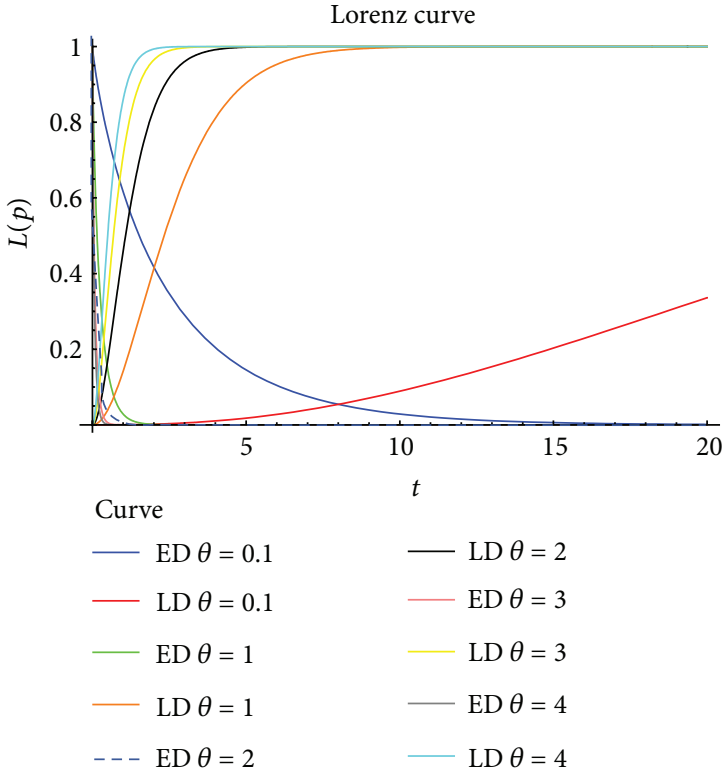

FIGURE 2: Lorenz curve graph.

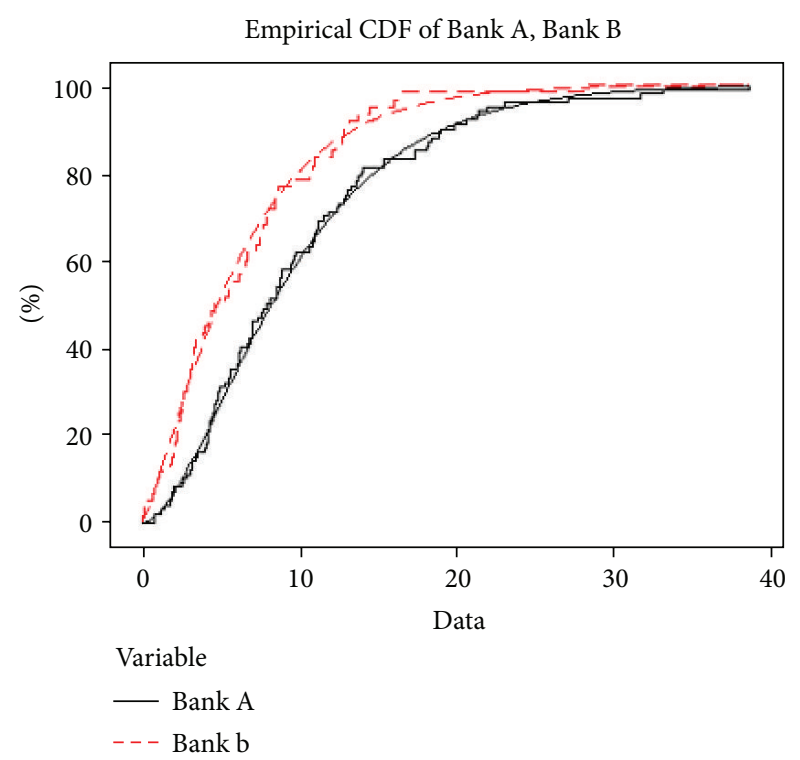

FIgURE 3: Empirical CDF of bank data.

For the Lindley distribution, the Lorenz curve is

$$
\begin{array}{r}
L(p)=1-\frac{\theta(\theta+1)(1-p)}{(\theta+2)(\theta+1+\theta t)}\left(\frac{2}{\theta}+1+2 t+\theta t+\theta t^{2}\right) \\
\text { where } p=F(t) .
\end{array}
$$

The comparison of Lorenz curve for exponential and Lindley distribution is given in Figures 2 and 3. Lindley distribution performance for Lorenz curve is better as compared to exponential distribution for different values of $\theta$. 
TABLE 19: BEs and respective PRs of stress and strength under different prior of real data where $R=0.354264$.

\begin{tabular}{lcccc}
\hline$\downarrow$ Loss function|prior $\rightarrow$ & UP & JP & LMP & GP \\
\hline SELF & $0.354572(0.001590)$ & $0.35578(0.001588)$ & $0.354518(0.001589)$ & $0.355719(0.001588)$ \\
WSELF & $0.350132(0.004439)$ & $0.351317(0.004436)$ & $0.35008(0.004438)$ & $0.351257(0.004432)$ \\
MSELF & $0.345899(0.958108)$ & $0.347017(0.957694)$ & $0.34585(0.957126)$ & $0.346961(0.957115)$ \\
PLF & $0.356806(0.004470)$ & $0.358004(0.004448)$ & $0.356754(0.004470)$ & $0.357944(0.004449)$ \\
SLLF & $0.352333(0.012639)$ & $0.353537(0.012664)$ & $0.35228(0.012637)$ & $0.353476(0.012663)$ \\
ELF & $0.350132(2.09262)$ & $0.351317(2.08584)$ & $0.35008(2.09292)$ & $0.351257(2.08618)$ \\
KLF & $0.352345(0.025358)$ & $0.353541(0.023508)$ & $0.352292(0.025354)$ & $0.353481(0.025307)$ \\
\hline
\end{tabular}

TABLE 20: BEs and respective PRs of mean residual life function under different prior of real data of Bank A.

\begin{tabular}{lcccc}
\hline$\downarrow$ Loss function|prior $\rightarrow$ & UP & JP & LMP & GP \\
\hline SELF & $8.40917(0.477926)$ & $8.45888(0.475225)$ & $8.40618(0.477931)$ & $8.45692(0.475245)$ \\
WSELF & $8.35267(0.056499)$ & $8.40204(0.056847)$ & $8.34973(0.056459)$ & $8.40007(0.056485)$ \\
MSELF & $8.29763(574.087)$ & $8.34567(584.203)$ & $8.29478(573.489)$ & $8.34375(583.797)$ \\
PLF & $8.43754(0.056738)$ & $8.48693(0.056088)$ & $8.43456(0.056735)$ & $8.48498(0.056124)$ \\
SLLF & $8.38079(0.006753)$ & $8.43049(0.006754)$ & $8.37781(0.006750)$ & $8.42852(0.006754)$ \\
ELF & $8.35267(4.24852)$ & $8.40204(4.26033)$ & $8.34973(4.24782)$ & $8.40007(4.23986)$ \\
KLF & $8.38088(0.013528)$ & $8.43041(0.013531)$ & $8.37791(0.013523)$ & $8.42845(0.013524)$ \\
\hline
\end{tabular}

\section{Stress and Strength Analysis for Lindley Distribution}

Let $X$ and $Y$ be two random variables such that $X$ represents "strength" and $Y$ "stress"; then, reliability of the stress and strength model is presented as

$$
R=P(Y<x)=\int_{-\infty}^{\infty} \int_{-\infty}^{x} f(x, y) d y d x
$$

where $P(Y<X)$ is a relationship which represents the probability that the strength exceeds the stress and $f(x, y)$ is joint p.d.f. of $X$ and $Y$ for example, receptor in human eye operates only if it is simulated by a source where magnitude $X$ is greater than a random lower threshold $Y$ for the eye, so here $R$ is the probability that the receptor operates. In mechanical reliability of a system $R$ gives the probability of a system failure, if the system fails whenever the strength is less than the applied stress.

This quandary has a long narration starting with the revolutionary work of Birnbaum [21] and Birnbaum and McCarty [22]. The stress strength was first introduced by Church and Harris [23]. A comprehensive handling of the different stress strength models can be found in outstanding monograph by Kotz et al. [24]. Some most fresh work on the stress strength model can be found in Kundu and Gupta [25, 26], Raqab and Kundu [27], Kundu and Raqab [28], Krishnamoorthy et al. [29], Eryilmaz [30], and the references cited therein. Recently, Al-Mutairi et al. [31] considered the stress and strength analysis of Lindley distribution using SELF but we are generalizing their work by considering different types of loss functions.

Suppose that $X$ and $Y$ are two independent Lindley rv's with respective parameters $\theta_{1}$ and $\theta_{2}$, then,

$$
\begin{gathered}
R=P(Y<X)=\int_{0}^{\infty} P(Y<X \mid Y=y) f(y) d y \\
=\int_{0}^{\infty} \int_{0}^{x} f(x, y) d y d x \\
R=\frac{\theta_{1}^{2}\left(\theta_{1}\left(1+\theta_{1}\right)+\left(1+\theta_{1}\right)\left(3+\theta_{1}\right) \theta_{2}+\left(3+2 \theta_{1}\right) \theta_{2}^{2}+\theta_{2}^{3}\right)}{\left(1+\theta_{1}\right)\left(1+\theta_{2}\right)\left(\theta_{1}+\theta_{2}\right)^{3}} .
\end{gathered}
$$

Remark. If $\theta_{1}=\theta_{2}=\theta$, then, $R=0.5$.

$$
\begin{array}{r}
I(x)=E\left[R=u\left(\theta_{1}, \theta_{2}\right)\right] \\
=\frac{\int_{\theta} u\left(\theta_{1}, \theta_{2}\right) \exp \left[l\left(\theta_{1}, \theta_{2}, \mathbf{x}\right)+g\left(\theta_{1}, \theta_{2}\right)\right] d \theta_{1} d \theta_{2}}{\int_{\theta} \exp \left[l\left(\theta_{1}, \theta_{2}, \mathbf{x}\right)+g\left(\theta_{1}, \theta_{2}\right)\right] d \theta_{1} d \theta_{2}} \\
\theta_{1}, \theta_{2}>0,
\end{array}
$$

where $u\left(\theta_{1}, \theta_{2}\right)=$ function of $\theta_{1}$ and $\theta_{2}$ only; $l\left(\theta_{1}, \theta_{2}, \mathbf{x}\right)=\log$ of likelihood; $g\left(\theta_{1}, \theta_{2}\right)=\log$ of prior of $\theta_{1}$ and $\theta_{2}$, can be evaluated as

$$
\begin{aligned}
& I(x)=u\left(\widehat{\theta_{1}}, \hat{\theta}_{2}\right) \\
& +0.5\left(\begin{array}{c}
\left(\hat{u}_{\theta_{1} \theta_{1}}+2 \bar{u}_{\theta_{1}} \hat{p}_{\theta_{1}}\right) \hat{\sigma}_{\theta_{\theta_{1}} \theta_{1}}\left(\hat{u}_{\theta_{1} \theta_{2}}+2 \widehat{u}_{\theta_{1}} \hat{p}_{\theta_{1}}\right) \hat{\sigma}_{\theta_{2} \theta_{1}} \\
+\left(\hat{u}_{\theta_{1} \theta_{2}}+2 \hat{u}_{\theta_{1}} \hat{p}_{\theta_{2}}\right) \hat{\sigma}_{\theta_{1} \theta_{2}}+\left(\hat{u}_{\theta_{2} \theta_{2}}+2 \hat{u}_{\theta_{2}} \hat{p}_{\theta_{2}}\right) \hat{\sigma}_{\theta_{2} \theta_{2}}
\end{array}\right)
\end{aligned}
$$

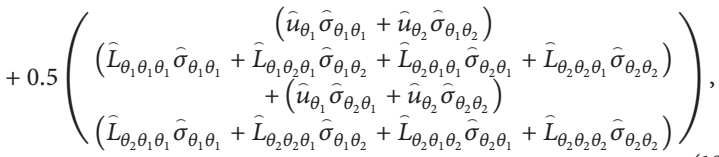


where $\widehat{\theta}_{j}=M L E$ of $\theta_{j}=\left(-(\bar{j}-1)+\sqrt{(\bar{j}-1)^{2}+8 \bar{j}}\right) /$ $2 \bar{j}, j=x, y ; \bar{x}>0 ; \hat{u}_{\theta_{i}}=\partial u\left(\widehat{\theta}_{i}\right) / \partial \hat{\theta}_{i} ; \hat{u}_{\theta_{i} \theta_{j}}=\partial^{2} u\left(\hat{\theta}_{i, j}\right) /$ $\partial \widehat{\theta}_{i, j}^{2} ; \hat{L}_{\theta_{i} \theta_{j} \theta_{i}}=\partial^{3} l\left(\widehat{\theta}_{i, j}\right) / \partial \hat{\theta}_{i, j}^{3} ; \quad \bar{p}_{\theta_{i}}=\partial g\left(\widehat{\theta}_{i, j}\right) / \partial \hat{\theta}_{i, j} ; \widehat{\sigma}_{\theta_{i} \theta_{j}}=$ $-1 / \widehat{L}_{\theta_{i} \theta_{j}} ; \hat{L}_{\theta_{i} \theta_{j}}=\partial^{2} l\left(\widehat{\theta}_{i, j}\right) / \partial \widehat{\theta}_{i, j}^{2} ; \hat{L}_{\theta_{i} \theta_{j} \theta_{i}}=\widehat{\sigma}_{\theta_{i} \theta_{j}}=0$ as both $x$ and $y$ are independent $R=0.026364$ (when $\theta_{1}=0.1$ and $\theta_{2}=1$ ); $R=0.0604$ (for $\theta_{1}=1$ and $\theta_{2}=9$ ) and $R=0.998771$ (when $\theta_{1}=9$ and $\theta_{2}=0.1$ ) (see Tables $9,10,11,12,13$, 14, and 15).

The Bayes estimates of stress and strength under SELF, MSELF, and ELF are underestimated. By comparing symmetric and asymmetric loss functions, it is noted that posterior risk of SELF is smaller than asymmetric loss functions. In case of asymmetric loss functions, WSELF and PLF have smaller posterior risk than other available loss functions.

Evaluating the performance of informative and noninformative priors, one can easily observe that informative priors have smaller posterior risk due to the availability of compact information. LM and gamma priors both have approximately the same behaviour depending upon the choice of hyperparameters value. More compact information will lead to correct hyperparameters which will lead to definitely better results and smaller posterior risk than noninformative priors. Although there are some depicts where informative priors have posterior risks greater than noninformative priors which is just due to random generation. Increasing sample size in case of SLLF has an inverse effect.

\section{Real Life Application}

Ghitany et al. [2] provide waiting times (in minutes) before service of 100 bank customers data set for Lindley distribution. They fitted both Lindley and exponential distributions (both have the same number of parameters) by method of maximized likelihood method and found Lindley distribution provides better fit. The data is given in Tables 16 and 17 .

We fit on both data sets Kolmogorov-Smirnov test and found that Lindley distribution is good fitted. The values of K-S test along $P$ value are given in Table 18.

The Bayes estimates of stress and strength reliability under different priors and their posterior risk are evaluated in Tables 19 and 20.

Since the Lindley distribution belongs to the exponential family so the natural conjugate prior is Gamma distribution.

The posterior risks of LMP and GP are approximately the same as compared to noninformative priors. There are some posterior risk values which are greater than noninformative priors. These are just due to hyperparameters value effect that is, more accurate values will lead to the smaller posterior risk. PLF and WSELF loss functions have smaller posterior risk as compared to other loss functions.

\section{Conclusion and Suggestions}

We consider the Bayesian analysis of the Lindley model via informative and informative priors under different loss functions. Based on posterior distribution, different properties, we conclude that informative priors (LMP, GP) performance approximately equal and have smaller posterior risk's as compared to the noninformative priors; also Jeffreys prior results are more precised than uniform prior. In other words, we can summarize result as

$$
\mathrm{GP}(\mathrm{PR}) \leq \mathrm{LMP}(\mathrm{PR})<\mathrm{JP}(\mathrm{PR})<\mathrm{UP}(\mathrm{PR}) .
$$

The choice of loss function as concerned, one can easily observe based on evidence (different properties as discussed above) that PLF, SLLF, and WSELF are suitable than other asymmetrical loss functions. One thing is common as we increase sample size posterior risk comes down. In future, this work can be extended using censored data.

\section{References}

[1] D. V. Lindley, "Fiducial distributions and Bayes' theorem," Journal of the Royal Statistical Society. Series B, vol. 20, pp. 102$107,1958$.

[2] M. E. Ghitany, B. Atieh, and S. Nadarajah, "Lindley distribution and its application," Mathematics and Computers in Simulation, vol. 78, no. 4, pp. 493-506, 2008.

[3] M. Sankaran, "The discrete Poisson-Lindley distribution," Biometrics, vol. 26, pp. 145-149, 1970.

[4] M. E. Ghitany and D. K. Al-Mutairi, "Estimation methods for the discrete Poisson-Lindley distribution," Journal of Statistical Computation and Simulation, vol. 79, no. 1, pp. 1-9, 2009.

[5] M. E. Ghitany, D. K. Al-Mutairi, and S. Nadarajah, "Zerotruncated Poisson-Lindley distribution and its application," Mathematics and Computers in Simulation, vol. 79, no. 3, pp. 279-287, 2008.

[6] H. Zamani and N. Ismail, "Negative binomial-Lindley distribution and its application," Journal of Mathematics and Statistics, vol. 6, no. 1, pp. 4-9, 2010.

[7] M. E. Ghitany, F. Alqallaf, D. K. Al-Mutairi, and H. A. Husain, "A two-parameter weighted Lindley distribution and its applications to survival data," Mathematics and Computers in Simulation, vol. 81, no. 6, pp. 1190-1201, 2011.

[8] H. Jeffreys, Theory of Probability, Oxford University Press, 3rd edition, 1964.

[9] A. K. Bansal, Bayesian Parametric Inference, Narosa Publishing House, New Delhi, India, 2007.

[10] M. Wasan, Parametric Estimation, McGraw-Hill, New York, NY, USA, 1970.

[11] K. Kanefuji and K. Iwase, "Estimation for a scale parameter with known coefficient of variation," Statistical Papers, vol. 39, no. 4, pp. 377-388, 1998.

[12] S. Ali, M. Aslam, and S. M. A. Kazmi, "A study of the effect of the loss function on Bayes Estimate, posterior risk and hazard function for Lindley distribution," Applied Mathematical Modelling, vol. 37, no. 8, pp. 6078-6078, 2013.

[13] I. Bayramoglu and S. Gurler, "On the mean residual life function of the k-out-of-n system with nonidentical components," in International Conference on Mathematical and Statistical Modeling in Honor of Enrique Castillo, pp. 28-30, Ciudad Real, Spain, June 2006.

[14] K. K. Govil and K. K. Aggarwal, "Mean residual life function for normal, gamma and lognormal densities," Reliability Engineering, vol. 5, no. 1, pp. 47-51, 1983. 
[15] B. Abdous and A. Berred, "Mean residual life estimation," Journal of Statistical Planning and Inference, vol. 132, no. 1-2, pp. 3-19, 2005.

[16] D. V. Lindley, "Approximate Bayesian methods," Trabajos de Estadistica Y de Investigacion Operativa, vol. 31, no. 1, pp. 223245, 1980.

[17] H. A. Howlader and A. M. Hossain, "Bayesian survival estimation of Pareto distribution of the second kind based on failurecensored data," Computational Statistics and Data Analysis, vol. 38, no. 3, pp. 301-314, 2002.

[18] R. Singh, S. K. Singh, U. Singh, and G. P. Singh, "Bayes estimator of generalized-exponential parameters under Linex loss function using Lindley's approximation," Data Science Journal, vol. 7, pp. 65-75, 2008.

[19] V. Preda, E. Panaitescu, and A. Constantinescu, "Bayes estimators of modified-Weibull distribution parameters using Lindley's approximation," WSEAS Transactions on Mathematics, vol. 9, no. 7, pp. 539-549, 2010.

[20] M. H. Gail and J. L. Gastwirth, "A scale-free goodness-of-fit test for the exponential distribution based on the Lorenz curve," Journal of the American Statistical Association, vol. 73, no. 364, pp. 787-793, 1978.

[21] Z. W. Birnbaum, "On a use of the Mann-Whitney statistic," in Proceedings of the 3rd Berkeley Symposium on Mathematical Statistics and Probability, vol. 1, pp. 13-17, University of California Press, Berkeley, Calif, USA, 1956.

[22] Z. W. Birnbaum and R. C. McCarty, "A distribution-free upper confidence bound for $\operatorname{Pr}(Y<X)$, based on independent samples of $X$ and $Y$," Annals of Mathematical Statistics, vol. 29, pp. 558-562, 1958.

[23] J. D. Church and B. Harris, "The estimation of reliability from stress strength relationships," Technometrics, vol. 12, pp. 49-54, 1970.

[24] S. Kotz, Y. Lumelskii, and M. Pensky, The Stress-Strength Model and Its Generalizations: Theory and Applications, World Scientific, River Edge, NJ, USA, 2003.

[25] D. Kundu and R. D. Gupta, "Estimation of $P(Y<X)$ for Weibull distributions," IEEE Transactions on Reliability, vol. 55, no. 2, pp. 270-280, 2006.

[26] D. Kundu and R. D. Gupta, "Estimation of $P(Y<X)$ for generalized exponential distribution," Metrika, vol. 61, no. 3, pp. 291-308, 2005.

[27] M. Z. Raqab and D. Kundu, "Comparison of different estimators of $P(Y<X)$ for a scaled Burr type $X$ distribution," Communications in Statistics: Simulation and Computation, vol. 34, no. 2, pp. 465-483, 2005.

[28] D. Kundu and M. Z. Raqab, "Estimation of $R=P(Y<X)$ for three-parameter Weibull distribution," Statistics and Probability Letters, vol. 79, no. 17, pp. 1839-1846, 2009.

[29] K. Krishnamoorthy, S. Mukherjee, and H. Guo, "Inference on reliability in two-parameter exponential stress-strength model," Metrika, vol. 65, no. 3, pp. 261-273, 2007.

[30] S. Eryilmaz, "On system reliability in stress-strength setup," Statistics and Probability Letters, vol. 80, no. 9-10, pp. 834-839, 2010.

[31] D. K. Al-Mutairi, M. E. Ghitany, and D. Kundu, "Inferences on stress-strength reliability from Lindley distribution," to appear in Communications in Statistics-Theory and Methods, available from http://home.iitk.ac.in/ kundu/lindley-ss.pdf. 

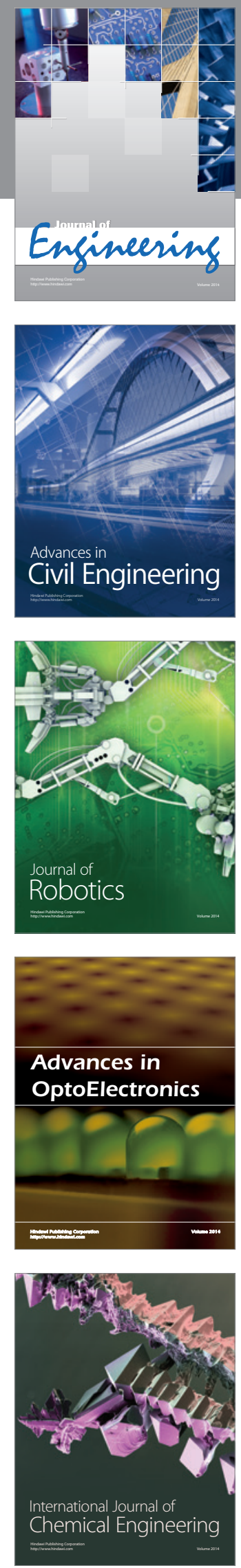

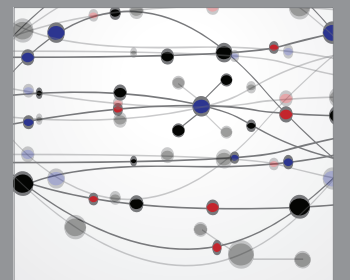

The Scientific World Journal
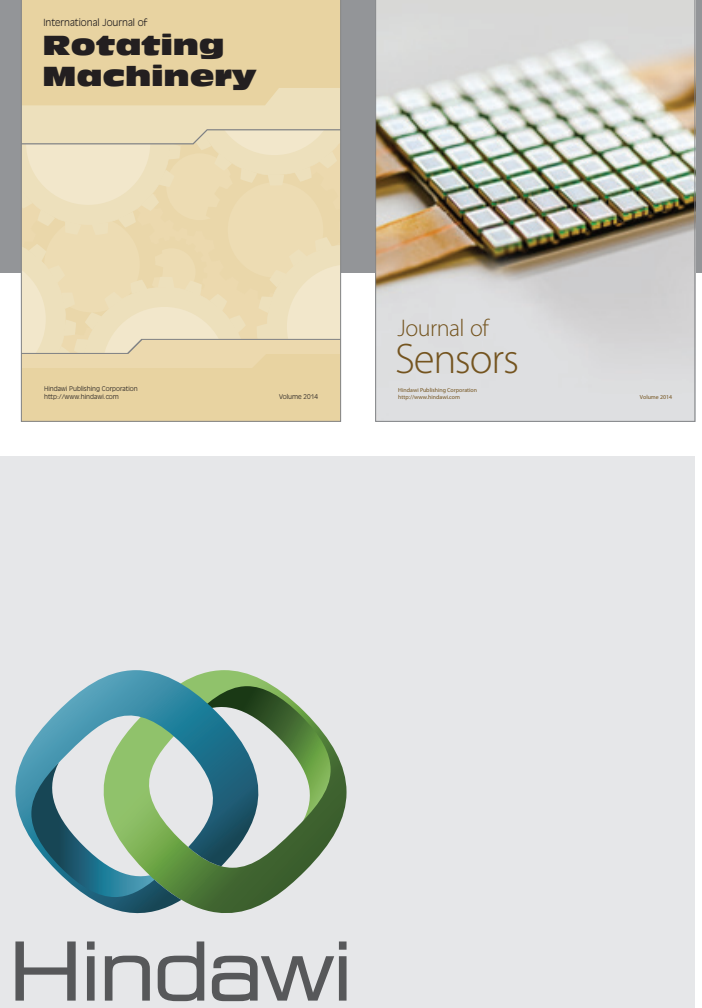

Submit your manuscripts at http://www.hindawi.com
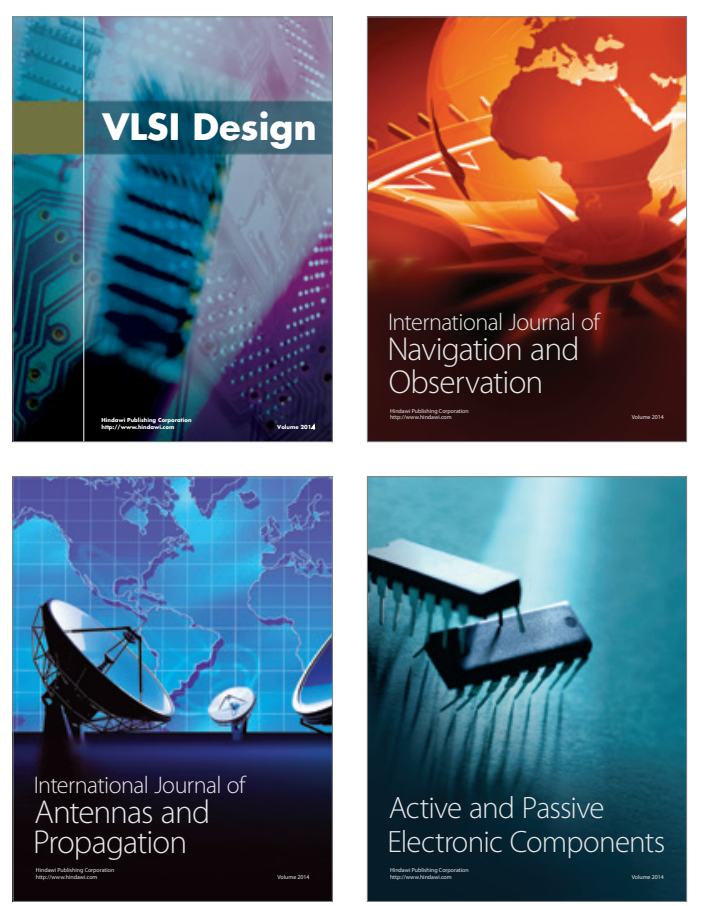
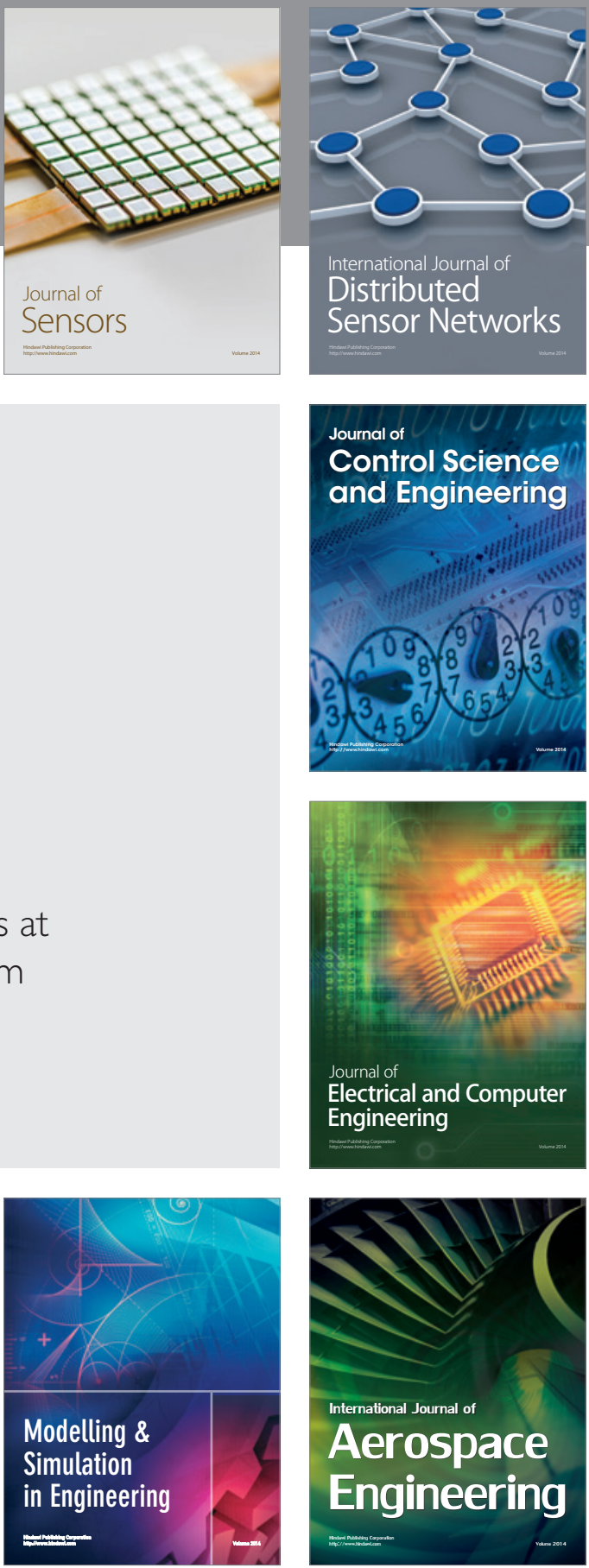

Journal of

Control Science

and Engineering
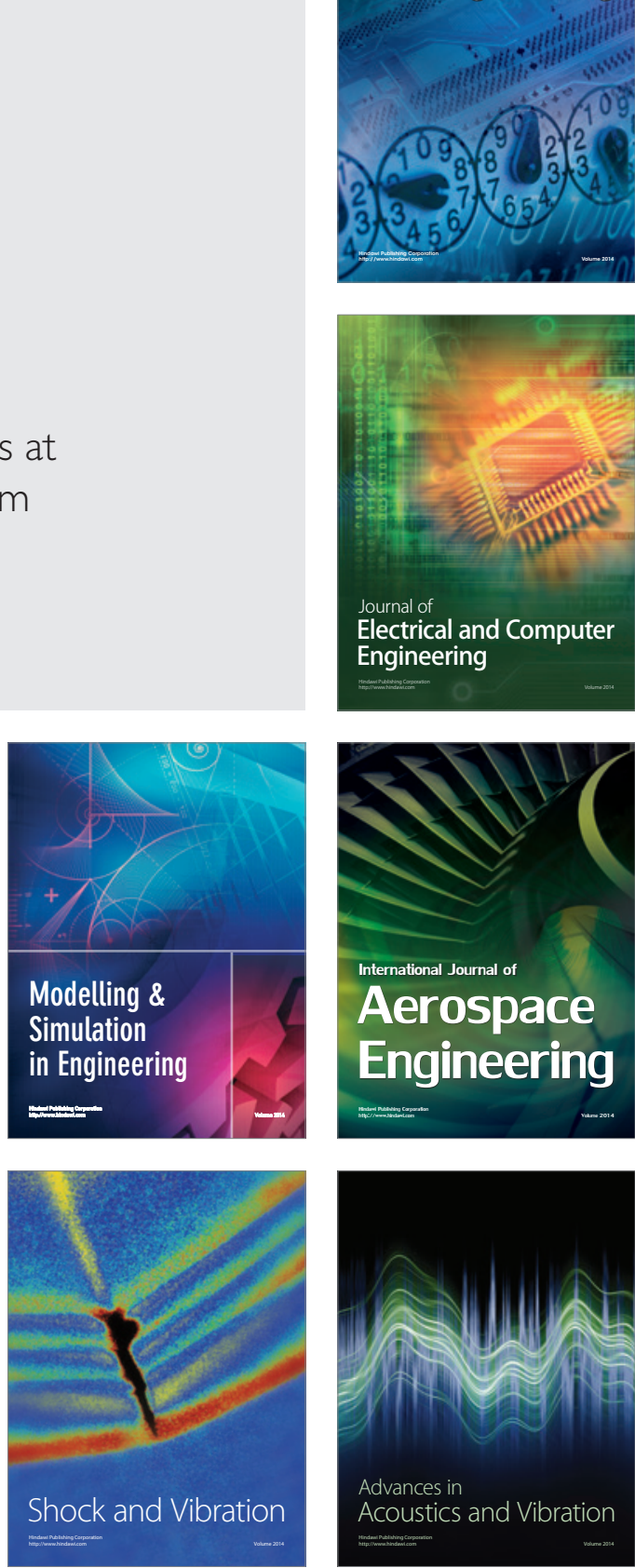